ARTICLE

https://doi.org/10.1038/s41467-019-09223-9

\title{
Lipidll interaction with specific residues of Mycobacterium tuberculosis PknB extracytoplasmic domain governs its optimal activation
}

Prabhjot Kaur ${ }^{1}$, Marvin Rausch (10) 2,3, Basanti Malakar ${ }^{1}$, Uchenna Watson ${ }^{4}$, Nikhil P. Damle ${ }^{1,6}$, Yogesh Chawla ${ }^{1,7}$, Sandhya Srinivasan ${ }^{5}$, Kanika Sharma ${ }^{5}$, Tanja Schneider ${ }^{2,3}$, Gagan Deep Jhingan ${ }^{5}$, Deepak Saini ${ }^{4}$ Debasisa Mohanty', Fabian Grein (1) ${ }^{2,3}$ \& Vinay Kumar Nandicoori (i) ${ }^{1}$

The Mycobacterium tuberculosis kinase PknB is essential for growth and survival of the pathogen in vitro and in vivo. Here we report the results of our efforts to elucidate the mechanism of regulation of $\mathrm{PknB}$ activity. The specific residues in the PknB extracytoplasmic domain that are essential for ligand interaction and survival of the bacterium are identified. The extracytoplasmic domain interacts with mDAP-containing Lipidll, and this is abolished upon mutation of the ligand-interacting residues. Abrogation of ligand-binding or sequestration of the ligand leads to aberrant localization of PknB. Contrary to the prevailing hypothesis, abrogation of ligand-binding is linked to activation loop hyperphosphorylation, and indiscriminate hyperphosphorylation of PknB substrates as well as other proteins, ultimately causing loss of homeostasis and cell death. We propose that the ligand-kinase interaction directs the appropriate localization of the kinase, coupled to stringently controlled activation of $\mathrm{PknB}$, and consequently the downstream processes thereof.

\footnotetext{
${ }^{1}$ National Institute of Immunology, Aruna Asaf Ali Marg, New Delhi 110067, India. ${ }^{2}$ Institute for Pharmaceutical Microbiology, University Hospital Bonn, University of Bonn, Bonn 53105, Germany. ${ }^{3}$ German Center for Infection Research (DZIF), Partner Site Bonn-Cologne, Bonn 53105, Germany. ${ }^{4}$ Department of Molecular Reproduction, Development and Genetics, Indian Institute of Science, Bengaluru 560012, India. ${ }^{5}$ Vproteomics, Valerian Chem Private Limited, Green Park Main, New Delhi 110016, India. ' $P$ Present address: BIOSS, Center for Biological Signaling Studies, University of Freiburg, Freiburg 79104, Germany. ${ }^{7}$ Present address: Department of Microbiology and Immunology, Weill Cornell Medical College, New York 10065 NY, USA. These authors contributed equally: Marvin Rausch, Basanti Malakar. Correspondence and requests for materials should be addressed to V.K.N. (email: vinaykn@nii.ac.in)
} 
$\mathrm{P}$ rotein phosphorylation has come forth as a preeminent circuitry regulating a vast number of physiological processes in the bacterial kingdom. A particular class of receptor-type serine-threonine kinase called PASTA (Penicillin binding proteins And Serine Threonine Associated) kinase is widespread across gram-positive firmicutes and actinomycetes and is known for its functions associated with bacterial cell growth $^{1}$. These protein kinases have an intracellular kinase domain, which shows sequence and structural homology to the eukaryotic serine/threonine kinases, and an extracytoplasmic (Ec) domain made up of varying number of PASTA domains. PASTA kinases are usually required by bacteria under stress conditions like nutrient starvation, antibiotic stress, biofilm formation etc., and are non-essential for their vegetative growth ${ }^{2}$. However, in the pathogenic bacterium Mycobacterium tuberculosis $(M t b)$ the PASTA kinase $\mathrm{PknB}\left(\mathrm{PknB}_{\mathrm{Mtb}}\right)$ is an essential gene $\mathrm{g}^{3-5}$ and is proposed to be one of the master regulators of serine/threonine phosphorylation-mediated signaling 6 .

The essential nature of $\mathrm{PknB}$ in mycobacteria stems from its ability to influence the activity of a large repertoire of substrates involved in cell wall synthesis, cell growth, cellular metabolism, transcription, and translation ${ }^{7}$. Over-expression or depletion of PknB impacts cellular morphology and survival of $M t b^{5,8}$, which suggests that the expression and activity of this kinase must be critically fine-tuned inside the bacterium. PknB levels are modulated under different conditions of mycobacterial growth: for instance its levels are down regulated during dormancy ${ }^{9}$ and nutrient starvation ${ }^{10}$ and are up regulated during exponential growth ${ }^{8}$ and resuscitation ${ }^{9}$. The dynamicity of $\mathrm{PknB}_{\mathrm{Mtb}}$ regulation implies that the receptor kinase actively monitors its environment and responds accordingly, in an effort to provide survival advantage. PknB is believed to respond to environmental signals through PASTA domain interactions with the specific ligand, identified to be non-crosslinked peptidoglycan (PG) fragments called muropeptides ${ }^{11}$. In line with this, purified PASTA domains of $\mathrm{PknB}_{\mathrm{Mtb}}$ interact and bind with a synthetic muropeptide containing isoglutamine (iGln) and meso-diaminopimelic acid (mDAP) residues at the second and third position of the stem peptide in vitro (Fig. 1a) ${ }^{11,12}$.

The prevailing hypothesis suggests that the interaction of the extracytoplasmic domain with the ligand results in the dimerization of intracellular kinase domain, which is required for the activation of the kinase through activation loop phosphorylations (Fig. 1b) ${ }^{13}$. The hypothesis is based on the front-to-front and back-to-back dimeric crystal structures of cytosolic kinase domain $^{14,15}$ and surface plasmon resonance-based in vitro binding experiments of PASTA domain with the muropeptides ${ }^{11}$. In consonance with this we have previously reported that the extracytoplasmic PASTA domains are indispensable for the function of $\mathrm{PknB}$ and survival of $M t b^{5}$. Deletion of the terminal PASTA domain (PASTA4) alone results in compromised survi$\mathrm{val}^{5}$, suggesting that it plays a leading role in kinase-ligand interactions. To date, the hypothesis with respect to $\mathrm{PknB}_{\mathrm{Mtb}}$ activation has not been tested in vivo. Here we set out to answer the following questions: (i) Is PASTA4 sufficient for $\mathrm{PknB}_{\mathrm{Mtb}}$ signaling?, (ii) What are the ligand binding residues in the extracytoplasmic domain?, (iii) What is the impact of abrogating ligand binding on the localization and activation of $\mathrm{PknB}$ ?, (iv) What are the physiological ligands that interact with PASTA domains? and (v) What is the impact of abrogating ligand binding on the phosphorylation of target substrates of PknB?

Here we identify ligand interacting residues and show that mutating these residues caused abolition of ligand binding. Abrogation of ligand binding triggers aberrant localization and hyperactivation of $\mathrm{PknB}$, which in turn results in hyperphosphorylation of both canonical and non-canonical downstream target substrates, eventually leading to cell death. Results suggest that interaction with the ligand is critical for appropriate localization and regulation of the kinase activity.

\section{Results}

PASTA4 and domain length are essential for PknB function. We have previously shown that the deletion of the PASTA4 domain (Fig. 1c; PknB-123) compromises the in vivo functionality of $\mathrm{PknB}^{5}$. The terminal PASTA domain of StkP, a Streptococcus pneumoniae ortholog of the $\mathrm{PknB}_{\mathrm{Mtb}}$, was demonstrated to be necessary and sufficient for it's signaling ${ }^{16}$. Thus we sought to determine the role of the terminal PASTA4 in the context of shorter total domain length. We employed previously described $M t b$ conditional mutant of $p k n B(R v \Delta B)$, wherein the native locus has been modified to bring its expression under pristinamycin inducible promoter, which allows the bacterium to grow efficiently in the presence of pristinamycin but not in its absence ${ }^{5,17}$ (Supplementary Fig. 1a). To assess the effect of PknB mutations, we generated wild type or mutant constructs in pNit-3F (Supplementary Fig. 1b), which could be induced with isovaleronitrile and owing to the presence of 3X-FLAG tag, the ectopically expressed PknB migrates slower compared with the endogenous protein (Supplementary Fig. 1c).

To investigate the role of PASTA4 as well as the domain length, we have generated PknB-234, lacking PASTA1 wherein the terminal PASTA4 is retained, and PASTA-1212, a chimera of appropriate domain length where PASTA1-2 were repeated (Fig. 1c). Western blot analysis confirmed the efficient depletion of $\mathrm{PknB}$ in the absence of pristinamycin as well as efficient expression of 3X-FLAG tagged wild-type and mutant proteins (Fig. 1d). On examining the ability of wild-type and mutant PknB proteins to complement the in vivo functionality of $\mathrm{PknB}$ (Fig. 1e), it was observed that vector-transformed $R v \Delta B$ showed significantly compromised survival in the absence of pristinamycin while the ectopic expression of $3 \mathrm{~F}-\mathrm{PknB}$ rescued the growth defects (Fig. 1e). Even though PknB-234 was marginally better compared to either PknB-123 or PknB-1212 ( 1.5 vs $2 \log$ fold) in rescuing the growth defects, growth was significantly compromised in all three strains when compared with the wild type (Fig. 1e). To assess the impact in an infection scenario, we evaluated the survival in differentiated THP1 cells (Fig. 1f). The data resembled the in vitro growth results, wherein PknB-234 showed slightly better survival as compared with PknB-1212 or PknB-123. These results suggest that while the PASTA4 domain is quite distinct and important, yet the appropriate length of the entire domain is also vital for efficient $\mathrm{PknB}_{\mathrm{Mtb}}$ function.

mDAP and iGln interacting residues influence the survival. The structure of the PASTA domains has been derived with the help of NMR as well as X-ray crystallography ${ }^{18,19}$. Nonetheless, the residues that are responsible for the interaction with the proposed muropeptide ligand have not yet been identified. As PASTA4 is critical for PknB functionality, we examined a possible role for it in ligand binding. As $\mathrm{PknB}$ is hypothesized to dimerize upon ligand binding ${ }^{14}$, we assumed that the PASTA domains interact with the muropeptide as a dimer. With the help of in silico molecular simulations, we identified a potential dimerization interface between the PASTA4 domains (Fig. 2a), which predominantly comprises of residues that are conserved across the PASTA4 domains of prokaryotic kinases (Supplementary Fig. 2). The initial simulation data suggested that ligand binding might be extending into the PASTA3 domain; hence, the dimeric PASTA3-4 domain was employed for further analysis. The ligand-binding domain majorly comprised of residues in the linker regions between PASTA3 and 4 domains (Fig. 2a \& 
a

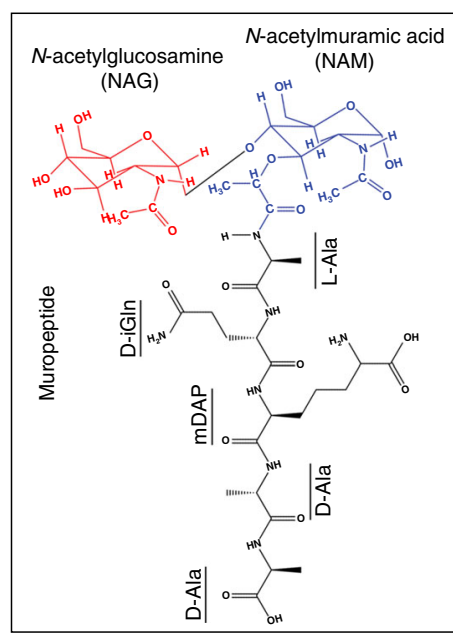

b

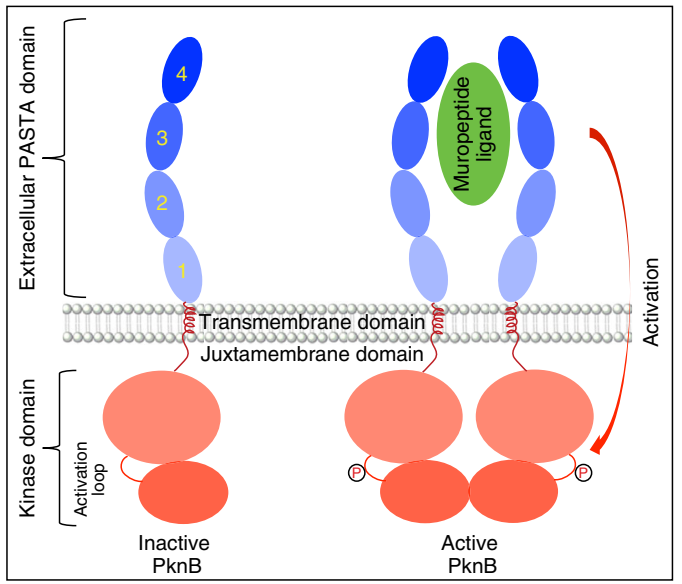

C

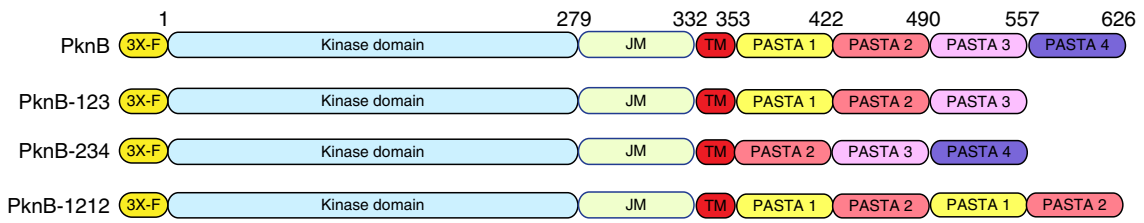

d

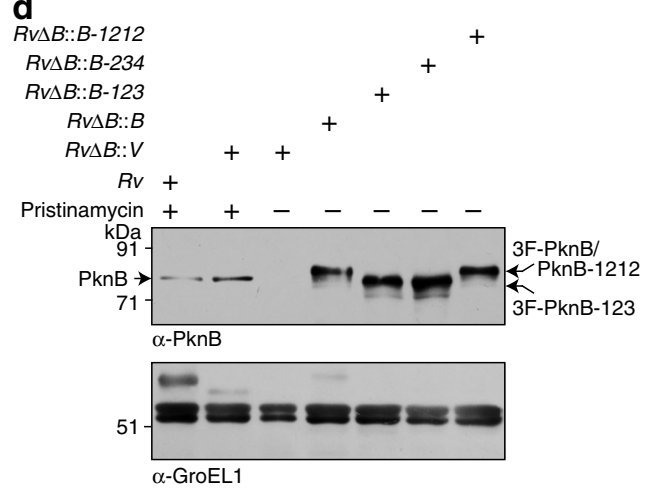

f

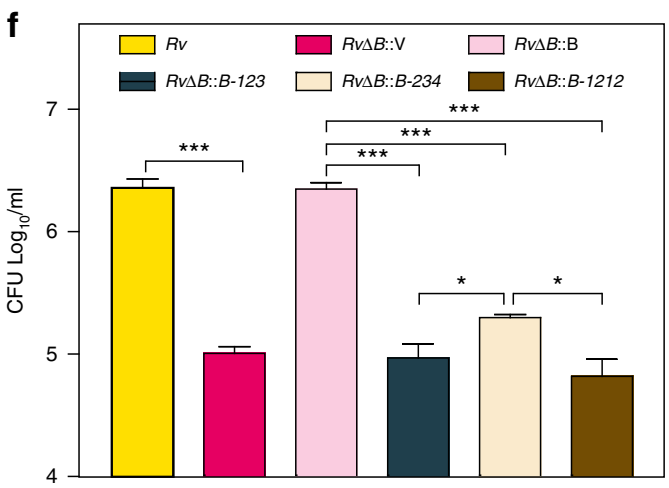

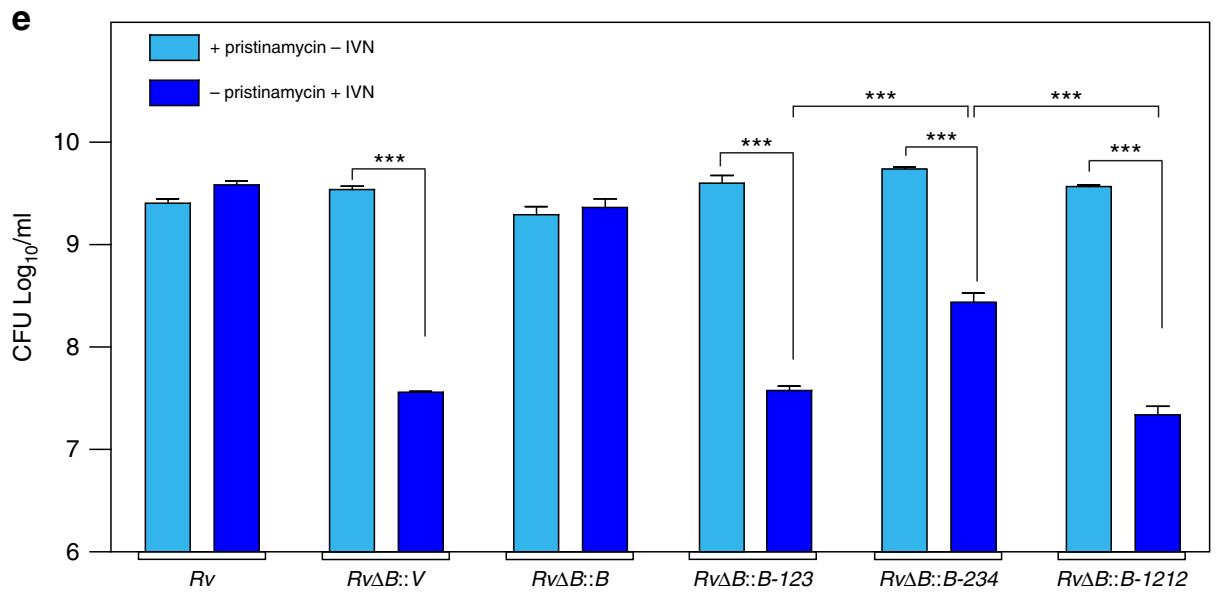

Supplementary Fig. 2). Data suggested that $\operatorname{Ser}_{556}$ and $\operatorname{Lys}_{557}$ (SK) interact with the carboxy terminal region of the iGln and $\mathrm{Asn}_{559}$ and $\mathrm{Gln}_{560}(\mathrm{NQ})$ interact with the mDAP residues in the muropeptide ligand (Fig. 2a \& Supplementary Fig. 2a). We set out to assess the impact of mutating these putative iGln and mDAP binding residues in modulating PknB functionality. We generated a PknB tetra mutant (PknB-GM), wherein all four putative ligand interacting residues have been mutated simultaneously (Fig. 2b). The $R v \Delta B$ strain was transformed with pNit-3F-PknB and pNit3F-PknB-GM and the expression of both proteins was confirmed (Fig. 2c). While the wild type could successfully rescue the phenotype both in vitro and ex vivo, the tetra mutant failed to do so (Fig. 2d, e). The growth phenotype did not vary even when the expression of $\mathrm{PknB}$ from pNit-3F constructs was not induced 
Fig. 1 PASTA4 and domain length are essential for PknB function. a Chemical structure of suggested muropeptide ligand for PknB. $\mathbf{b}$ Schematic outline of prevailing hypothesis vis-a-vis $\mathrm{PknB}$ activation, wherein the ligand binding is proposed to result in dimerization and phosphorylation of the activation loop residues in the intracellular kinase domain. $\mathbf{c}$ Schematic representation of full-length PknB, PknB-PASTA domain deletion mutants, and the chimera. $\mathbf{d}$ Rv $\Delta B$ strain was electroporated with pNit-3F, pNit-3F-PknB, pNit-3F-PknB-123, pNit-3F-PknB-234, and pNit-3F-PknB-1212 to generate $R v \Delta B:: V, R v \Delta B:: B, R v \Delta B:: 123$, $R v \Delta B:: 234$, and $R v \Delta B:: 1212$. Whole-cell lystaes (WCLs) were prepared from cultures initiated at $A_{600}$ of $\sim 0.05$ and grown for 5 days in the presence or absence of of pristinamycin. IVN was added in the absence of pristinamycin to the cultures to induce the expression of episomal copy. Ten microgram each of WCLs were resolved on $8 \%$ SDS-PAGE, transferred to nitrocellulose membrane, and western blotted with $\alpha$-PknB and $\alpha$-GroEL1 antibodies. The endogenous and episomally expressed $\mathrm{PknB}$ are indicated by arrow. e $R v$ and $R v \Delta B$ transformants were inoculated at initial $\mathrm{A}_{600}$ of $\sim 0.05$ and grown in the presence or absence of pristinamycin. IVN $(0.2 \mu \mathrm{M})$ was added in the absence of pristinamycin to the cultures to induce the expression of episomal copy. After 6 days of in vitro growth, Colony forming Units (CFU) were enumerated by plating appropriate dilutions on $7 \mathrm{H} 11$ agar plates containing pristinamycin. Data is representative of three biologically independent experiments with each experiment being performed in triplicates. $\mathbf{f} R v$ and $R v \Delta B$ transformants grown in the presence of pristinamycin till $A_{600}$ of $\sim 0.6-1.0$ were washed thrice with PBS to remove inducer. These cultures were used to infect differentiated THP-1 human macrophages at 1:10 M.O.I. IVN was added in the media to induce the expression of episomal copy and CFUs were evaluated $72 \mathrm{~h}$ post infection (p.i). Data are representative of one of the two biologically independent experiments and each experiment was performed in triplicates. Statistical analysis was performed with the unpaired $t$-test using Graphpad software. Data shown represents mean + standard deviation (SD). ${ }^{\star \star} p<0.005$, ${ }^{\star \star \star} p<0.0005$. Source data are provided as a Source Data file

with isovaleronitrile, suggesting that the results are not due to overexpression artifacts (Supplementary Fig. 2b, c). Thus, putative iGln and mDAP interacting residues in the PASTA domain of $\mathrm{PknB}$ seem to be necessary for its functionality.

iGln/mDAP interacting residues are independently essential. Next we sought to assess the impact of individually mutating the amino acid pairs, which we believed to interact with iGln or mDAP residues. Hence we generated $P k n B-G$ and $P k n B-M$, wherein SK or NQ residues were mutated to AA or DE residues, respectively (Fig. 3a). Western blot showed efficient expression of 3F-PknB, 3F-PknB-G, and 3F-PknB-M in the complemented strains (Fig. 3b). Compared with the tetra mutant (Fig. 2d), strains complemented with either PknB-G or PknB-M showed partial growth defects, albeit to different extents (Fig. 3c, e). PknB-G (Rv $\Delta B:: B-G)$ showed one and half log-fold poorer survival compared with $\mathrm{PknB}(R v \Delta B:: B)$, while it showed $\sim 3 \log$ folds better survival compared with the control $(R v \Delta B:: V)$ (Fig. 3c). On the other hand, PknB-M (Rv $\Delta B:: B-M)$ showed 3 log fold lower survival compared with $R v \Delta B:: B$ and one and half log-fold better survival compared with the control (Fig. 3e). These results suggested that mDAP interacting residues play a more critical role compared with iGln interacting residues. However in a THP1 infection model (Fig. 3d, f), both the mutants were equally abrogated and showed defects similar to the tetra mutant (Fig. 2e). THP1 infection experiment performed at lower MOI (1:4) showed similar defects as those observed at higher MOI (1:10) (Figs 2e and 3d, f). Data suggests that both iGln and mDAP interacting residues are individually critical for $\mathrm{PknB}$ functionality, especially during the ex vivo infection scenario where even marginal perturbations in ligand binding are not endured.

Mutations in the PknB-Ec abrogate its binding to LipidII. PG synthesis at the poles and septum region involves two stages. In the first stage, which happens in the cytoplasm, LipidII is synthesized from UDP-GlcNAc precursor by sequential action of multiple Mur family enzymes ${ }^{20}$. LipidII is composed of $\mathrm{N}$-acetylglucosamine (NAG)- $N$-acetylmuramic acid (NAM)-pentapeptide (stem peptide) connected to the membrane anchored decaprenyl phosphate through a pyrophosphate link (Supplementary Fig. 3a). LipidII is then translocated/flipped into the periplasmic region, which provides the NAG-NAM-pentapeptide moieties to the growing PG layer ${ }^{20}$. The nature of the lipid moiety, the amino acids in the stem peptides and modification in the NAG and NAM sugars, vary from species to species ${ }^{21}$. The Staphylococcus aureus $\mathrm{PknB}$ ortholog $\left(\mathrm{PknB}_{s a}\right)$ has been shown to interact strongly with LipidII through PASTA domains (1:2 molar ratio of LipidII: $\left.\mathrm{PknB}_{s a}\right)^{22}$. Thus we sought to explore the possibility of an interaction between $\mathrm{PknB}_{\mathrm{Mtb}}$ and LipidII. Unlike $S$. aureus, wherein the LipidII stem peptide contains a lysine residue at the third position, the LipidII in $M t b$ carries mDAP at the analogous position in the stem peptide ${ }^{21}$ (Supplementary Fig. 3a).

It is apparent from the data presented in Figs. 2 and 3 that PASTA linker mutants were defective in rescuing PknB function, which may be due to major conformational changes. To assess the conformation changes, His-tagged $\mathrm{PknB}$ and the mutants of extracytoplasmic PASTA domains (PknB-Ec) were purified and $\mathrm{CD}$ analysis was performed (Fig. $4 \mathrm{a}-\mathrm{c}$ ). CD analysis indicated absence of gross conformational changes in the secondary structure (Fig. $4 \mathrm{a}-\mathrm{c}$ ). Next we sought to examine the interaction between the PknB-Ec (Fig. 4d) and lysine- or mDAP-containing LipidII molecules (Supplementary Fig. 3b). When mDAPcontaining LipidII was used in the assay, there was no detectable LipidII upon extraction at 4:1 molar ratio of PknB-Ec:LipidII, indicating a strong interaction (Fig. 4e). This interaction could be abrogated upon treating the complex with trypsin, which would degrade the protein (Fig. 4e). Interestingly, lysine-containing LipidII could not be sequestered into a complex by PknB-Ec even at a molar ratio of 8:1 (protein:LipidII; Fig. 4e). We then evaluated the ability of mutants to bind with mDAP-LipidII. It is evident from the data presented in Fig. $4 \mathrm{f}$ that neither double nor the tetra mutant showed any binding with the mDAP-LipidII (Fig. 4d, f). The results show that the extracytoplasmic domain of $\mathrm{PknB}_{M t b}$ binds specifically with mDAP-containing LipidII and the binding is abrogated upon mutating ligand-interacting residues.

Abrogation of ligand binding perturbs localization of PknB. Nascent PG biosynthesis takes place at the poles and mid cell (septum) regions ${ }^{23}$ and hence the precursors such as LipidII and muropeptides are anticipated to be concentrated at these niches. Interestingly, $\mathrm{PknB}$ also localizes to both polar and mid-cell regions and the extracytoplasmic PASTA domains govern the localization $^{11}$. Thus the rational supposition would be that the localization of $\mathrm{PknB}$ is probably dictated by the interaction between PASTA domain and PG precursors. However, the hypothesis has not been tested till date, perhaps due to the nonavailability of PknB mutants that fail to interact with the ligand. We examined the localization of GFP-PknB and GFP-PknB-GM mutants in $M$. smegmatis $p k n B$ conditional mutant $\left(m c^{2} \Delta B\right)$, following the reasoning that in the absence of endogenous $\mathrm{PknB}$; GFP-PknB or GFP-PknB-GM would be the sole PknB in the cell 
a

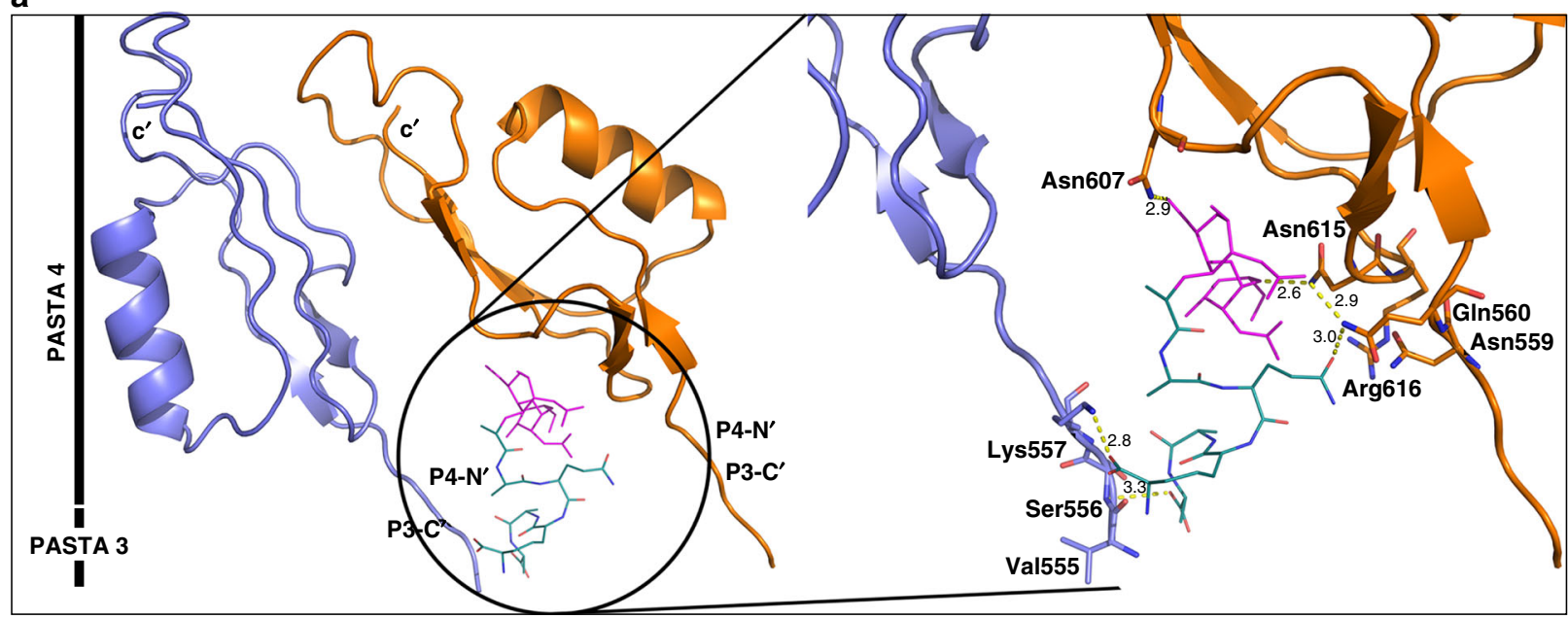

b
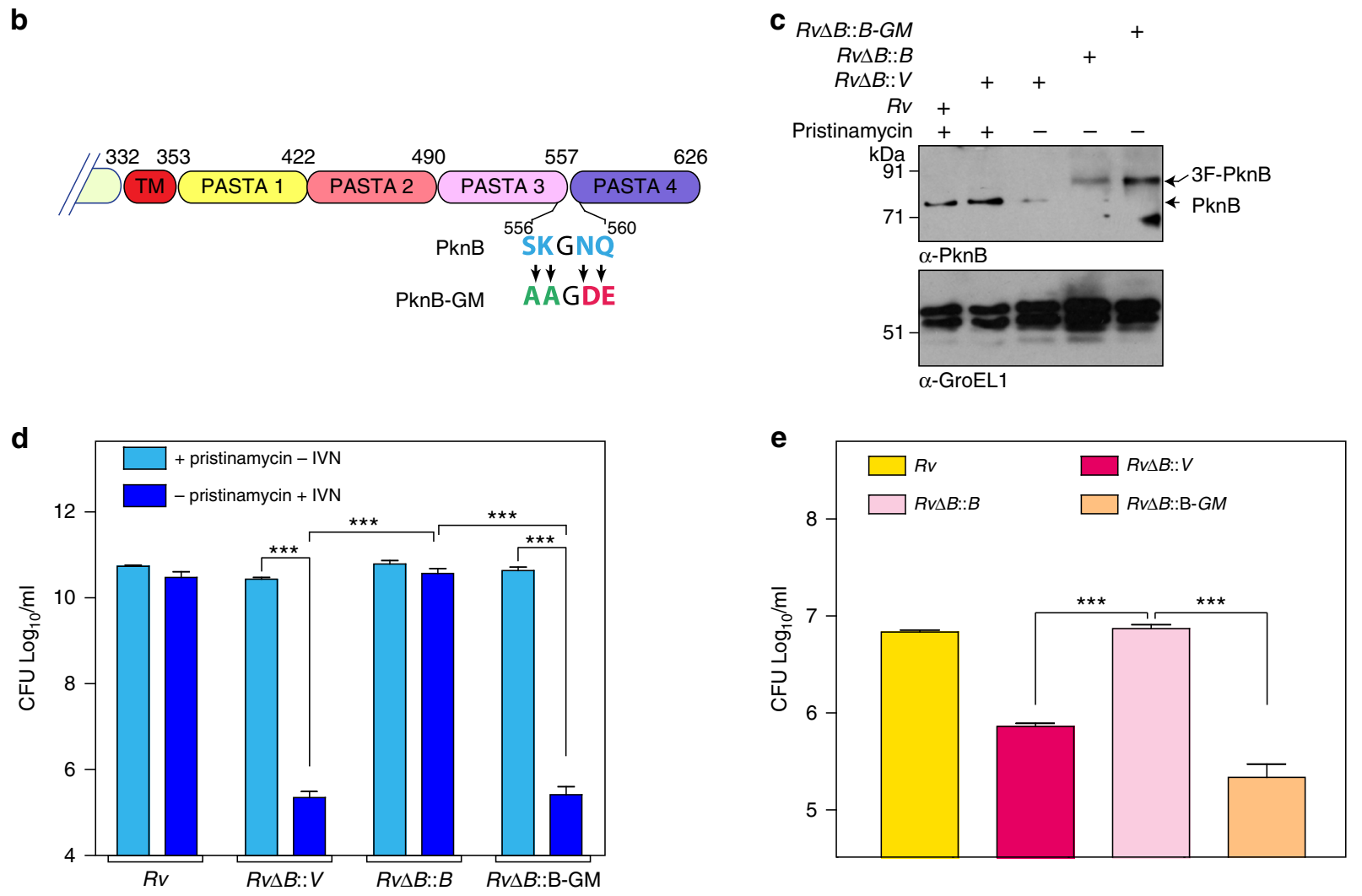

Fig. 2 mDAP and iGIn interacting residues influence the survival. a Model of Mtb PknB PASTA3-4 dimer in complex with the muropeptide ligand. Inset panel shows a magnified view of the interactions between the MDAP and iGIn residues of the muropeptide with polar amino acids between the PASTA3 and 4 domains of PknB. $\mathbf{b}$ Schematic representation of extra-cytoplasmic PASTA domain of PknB and the residues in the PASTA3-4 linker region suggested to be involved in interaction with iGln (SK) and mDAP (NQ) residues in the muropeptide. The mutations introduced in the linker region to generate PknBGM are indicated. c Western blot analysis of WCLs prepared from $R v$ and $R v \Delta B$ transformants. The transformants were seeded at $A_{600}$ of $\sim 0.05$ in the presence of pristinamycin or IVN as indicated for 5 days and WCLs were resolved on 8\% SDS-PAGE and probed with $\alpha$-PknB and $\alpha$-GroEL1 antibodies. d Cultures of $R v$ and $R v \Delta B$ transformants were initiated at $A_{600}$ of $\sim 0.05$ and grown in the presence of $1.5 \mu \mathrm{g} / \mathrm{ml}$ pristinamycin or $0.2 \mu \mathrm{M}$ IVN as indicated for 6 days. CFUs were evaluated by plating appropriate dilution on $7 \mathrm{H} 11$ agar plates containing pristinamycin. Data are representative of the three biologically independent experiments and each experiment was performed in triplicates. e Human monocytic cell line THP- 1 was differentiated to macrophages and was infected at 1:10 M.O.I. with $R v$ and $R v \Delta B$ transformants grown to $A_{600}$ of $\sim 0.6-1.0$ in the presence of pristinamycin. IVN was added in the media to induce the expression of episomal copy and CFUs were enumerated $72 \mathrm{~h} \mathrm{p.i.} \mathrm{Data} \mathrm{are} \mathrm{representative} \mathrm{of} \mathrm{one} \mathrm{of} \mathrm{the} \mathrm{two} \mathrm{biological} \mathrm{replicates}$ and each experiment was performed in triplicates. Data represents mean + SD. Statistical analysis was performed with the unpaired $t$-test using Graphpad software. ${ }^{\star \star} p<0.0005$. Source data are provided as a Source Data file 
a

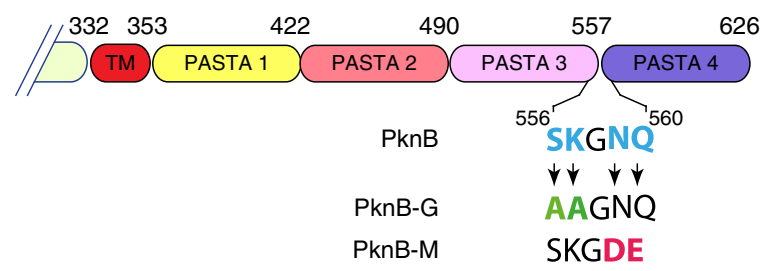

C

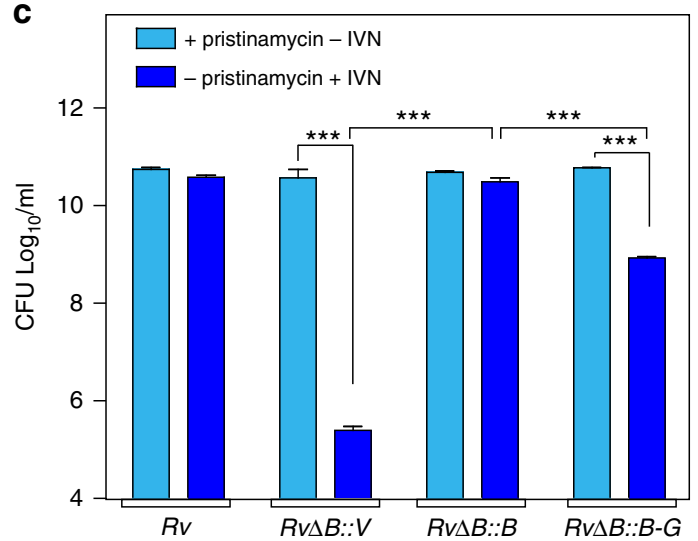

e

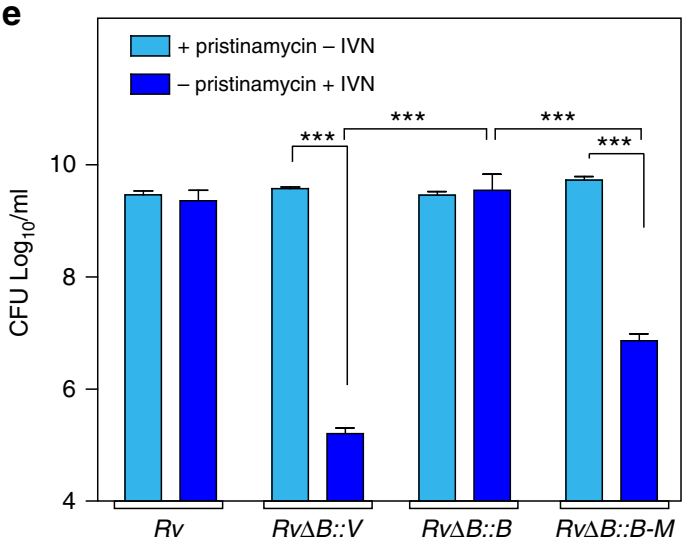

b

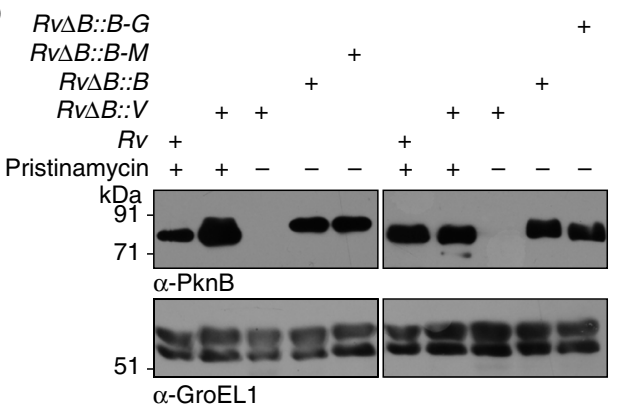

d

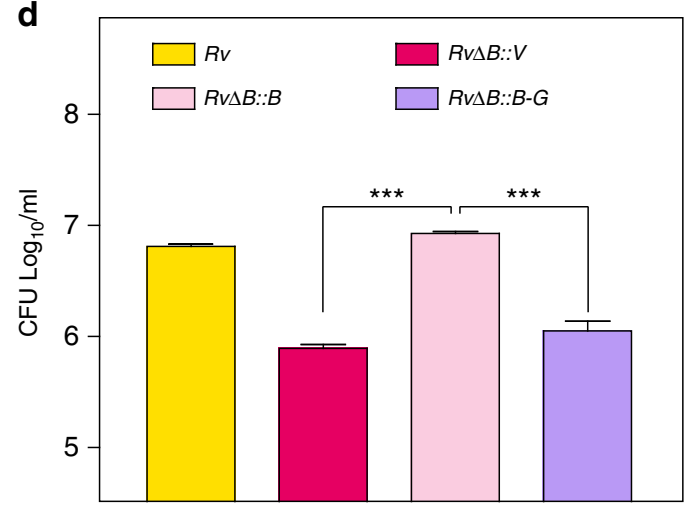

$\mathbf{f}$

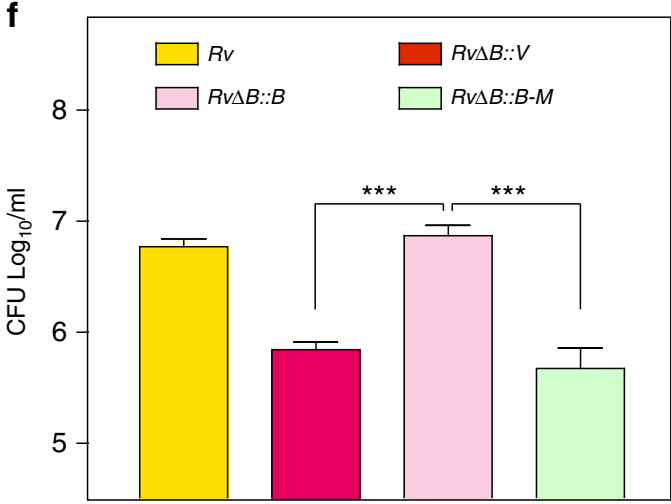

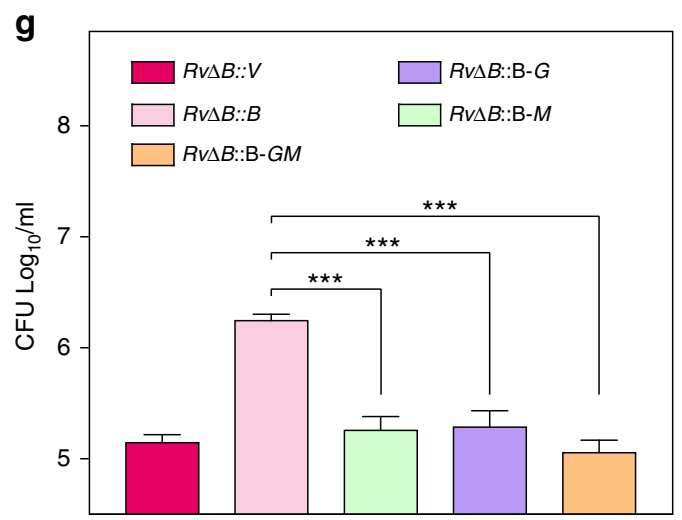

and hence phenotypic impact in terms of localization can be clearly visualized. Although GFP-PknB shows a strong punctate distribution at the polar and mid cell regions, GFP-PknB-GM mutant shows aberrant localization throughout the cell (Fig. 5a), suggesting that abrogation of ligand binding results in aberrant localization. Quantification of pole/septa vs. aberrant localization drives home the message (Fig. 5b). If this was indeed accurate, the converse experiment wherein the ligand is sequestered should also result in aberrant localization. Thus, we asked what would happen to PknB localization when LipidII is sequestered into a complex with antibiotic "nisin", which binds through the pyrophosphate group resulting in membrane pore formation and 
Fig. $3 \mathrm{iGln}$ or mDAP interacting residues are independently essential. a Schematic outline of the PknB and the PknB-mutants. The mutations introduced in the linker region to abrogate either iGln (SK $\rightarrow \mathrm{AA})$ or $\mathrm{mDAP}(\mathrm{NQ} \rightarrow \mathrm{DE}$ ) binding are indicated. $\mathbf{b}$ WCLs prepared from cultures of $R v$ and $R v \Delta B$ transformants inoculated at $A_{600}$ of $\sim 0.05$ in the presence of pristinamycin or IVN and grown for 5 days were probed with $\alpha$-PknB and $\alpha$-GroEL1 antibodies. c, e $R v$ and $R v \Delta B$ transformants were inoculated at initial $A_{600}$ of $\sim 0.05$ and grown for 6 days in the presence of pristinamycin or IVN. CFUs were enumerateed on $7 \mathrm{H} 9$ plates containing pristinamycin. Data are representative of the three biologically independent experiments with each experiment being performed in triplicates. d, f, $\mathbf{g}$ Differentiated THP-1 cells were infected at 1:10 (d, f) or 1:4 (g) M.O.I. with $R v$ and $R v \Delta B$ transformants grown to $A_{600}$ of $\sim 0.6-1.0$ in the presence of pristinamycin. Pristinamycin was removed from cultures prior to infection by washing with PBST 80 and PBS. IVN was added in the media to induce the expression of episomal copy and CFUs were enumerated $72 \mathrm{~h}$ p.i. Data are representative of one of the two biological experiments and each experiment was performed in triplicates. Data represents mean + SD. Statistical analysis was performed with the unpaired $t$-test using Graphpad software. ${ }^{\star \star \star} p<0.0005$. Source data are provided as a Source Data file

eventual cell death ${ }^{24-26}$. The antibiotic isoniazid (INH) that does not interact with LipidII was used as the control (Fig. 5c). Although we observed distinct puncta with $m c^{2} \Delta B:: G F P-B$ in the absence of any antibiotic or in the presence of INH, aberrant number/mislocalized puncta were observed ( $>3$ ) upon nisin treatment (Fig. 5c). Quantification showed a significant increase in the number of cells with more than 3 puncta or localization all through the cell (Fig. 5d). These results suggest that LipidII is likely to be the major intracellular ligand of $P \mathrm{knB}$, and importantly, ligand binding is essential for the appropriate localization. Since the PknB-GM fails to interact with the ligand and does not localize to pole and septum, we reasoned that it might impact the diffusion dynamics. We investigated the rate of recovery of GFP$\mathrm{PknB}$ and GFP-PknB-GM upon photobleaching by performing FRAP (Fluorescence Recovery After Photobleaching) experiments (Fig. 5e). Although GFP-PknB showed relatively slower recovery $\left(t_{1 / 2}=111 \mathrm{~s}\right)$, GFP-PknB-GM mutant showed substantially higher dynamicity with $t_{1 / 2}=90.56 \mathrm{~s}$ (Fig. 5f), suggesting that ligand binding also plays a role in modulating molecular dynamicity.

PknB-GM is hyper-phosphorylated in the activation loop. If the prevailing hypothesis with respect to the activation of $\mathrm{PknB}$ shown in Fig. $1 \mathrm{~b}$ is accurate, abrogation of ligand binding should result in decreased phosphorylation of the activation loop residues. PknB is auto-phosphorylated at the T171 and T173 residues within the activation loop in vitro, and mutating these residues significantly diminishes its activity ${ }^{27}$, (Fig. 6a). PknB is also phosphorylated in vitro in the juxtamembrane domain ${ }^{27,28}$, (Fig. 6a); however, there are no reports about the influence of these phosphorylations its function. To scrutinize the roles of T171 and T173 residues, we generated PknB-TATA (T171A, T173A) mutant (Supplementary Figs. 4a and 6a), and the activity was analyzed using GarA as the substrate (Supplementary Fig. $4 \mathrm{~b}-\mathrm{d})$. In agreement with the previous report ${ }^{27}$, the PknBTATA mutant showed significantly compromised activity (Supplementary Fig. 4c, d). To examine their in vivo role, $\mathrm{PknB}$ or PknB-TATA clones made using integrative vector as backbone were electroporated into $R v \Delta B$ cells. The expression of $\mathrm{PknB}$ and PknB-TATA from the integrated construct was comparable to that of inducible PknB at the native locus (Fig. 6b). Compared with $\operatorname{Rv} \Delta B:: V, \operatorname{Rv} \Delta B:: B-T A T A$ showed better survival; nonetheless, the mutant was significantly compromised when compared with $\operatorname{Rv} \Delta B:: B$, indicating the essentiality of activation loop phosphoryaltions (Fig. 6c). Importantly, in the ex vivo model of infection, both $\operatorname{Rv} \Delta B:: V$ and $\operatorname{Rv} \Delta B:: B-T A T A$ were found to be equally compromised (Fig. 6d).

To determine the extent of activation loop and juxtamembrane phosphorylations, we resorted to isobaric TMT labeling (Fig. 6e). Western blot and quantitative mass spectrometry analysis of total proteome demonstrated depletion of $\mathrm{PknB}$ and similar expression of 3F-PknB and 3F-PknB-GM (Fig. 6f, g). However, the levels of $P k n B$ protein in $\operatorname{Rv} \Delta B:: B \& \operatorname{Rv} \Delta B:: B-G M$ were $\sim 1.2-1.5\left(\log _{2}\right)$ fold higher compared with $\operatorname{PknB}$ in $\operatorname{Rv} \Delta B$ (+pristinamycin; Fig. 6g; Supplementary Table 1a). Intriguingly, contrary to the current belief, we observed hyperphosphorylation of both activation loop and juxtamembrane domain in the 3F-PknB-GM mutant compared with 3F-PknB (Fig. 6h \& Supplementary Table 1b). Although the role of activation loop phosphorylation is known, the role of juxtamembrane phosphorylation needs further investigation. Thus the data suggests that abrogation of ligand binding results in hyper phosphorylation of $\mathrm{PknB}$, strongly suggesting that the ligand binding plays a regulatory role.

PknB-GM hyperphosphorylates specific \& non-specific targets. We identified a total of 632 (Supplementary Fig. 6a \& Supplementary Data 1) and 258 (Supplementary Fig. 6b) proteins in the proteomic and phosphoproteomic samples, respectively. Of the 258 phosphoproteins, 242 had common phosphopeptides (total $=390$ phosphopeptides) in all three replicates. Among them, 147 proteins encompassing 257 phosphopeptides were present in both, proteomic and phosphoproteomic samples (Supplementary Fig. 6c \& Supplementary Data 2). The intensity ratios of these 257 phosphopeptides were normalized with respect to the wholeprotein intensity ratios (Supplementary Data 1) and the mean normalized ratios (Supplementary Data 3) were converted to $\log _{2}$ values (Supplementary Table 2) and plotted as a heatmap (Fig. 7a). The 147 phosphoproteins were distributed across all the functional categories (Fig. 7b) with a majority of the phosphorylations on threonine $(68 \%)$, followed by serine $(\sim 23 \%)$ and tyrosine $(\sim 9 \%)$, residues, a trend universal to all mycobacterial phosphoproteomic studies ${ }^{29-31}$. Among the 257 phosphopeptides, a total of 111 phosphopeptides mapping to 73 different proteins (which included multiple well-characterized substrates such as Ef-Tu, Wag31, and HupB) $)^{8,32,33}$ were potentially the products of PknB-mediated phosphorylation (Fig. 7b, c; Supplementary Table 3a). Recently, Carette et al. have identified 46 potential targets of $\mathrm{PknA}$ and $\mathrm{PknB}$ with the help of a specific inhibitor $^{34}$. Nine of these identified targets were found to be among the 73 potential targets of $\mathrm{PknB}$ identified in this study (Supplementary Table 3b). Interestingly, we also identified 8 putative PknB-dependent tyrosine phosphorylated peptides (Fig. 7d; Supplementary Table 4a, b), in agreement with a previous study ${ }^{35}$ where $\mathrm{PknB}$ was suggested to be a dual specificity kinase.

The analysis of the activation loop phosphorylations in $\mathrm{PknB}$ suggested that PknB-GM is hyperphosphorylated (Fig. 6h), which led us to infer it to be a hyperactive kinase. In line with this prediction, complementation with the PknB-GM mutant seems to have resulted in the remarkable hyperphosphorylation of cellular proteins (Fig. 7a; Supplementary Table 2). The data in Fig. 7a can be further divided into three clusters (Supplementary Table 4c): cluster 1 where the phosphorylation is unperturbed by depletion of $\mathrm{PknB}$ as well as complementation with ectopic expression of $\mathrm{PknB}$ or $\mathrm{PknB}-\mathrm{GM}$; cluster 2 representing direct 

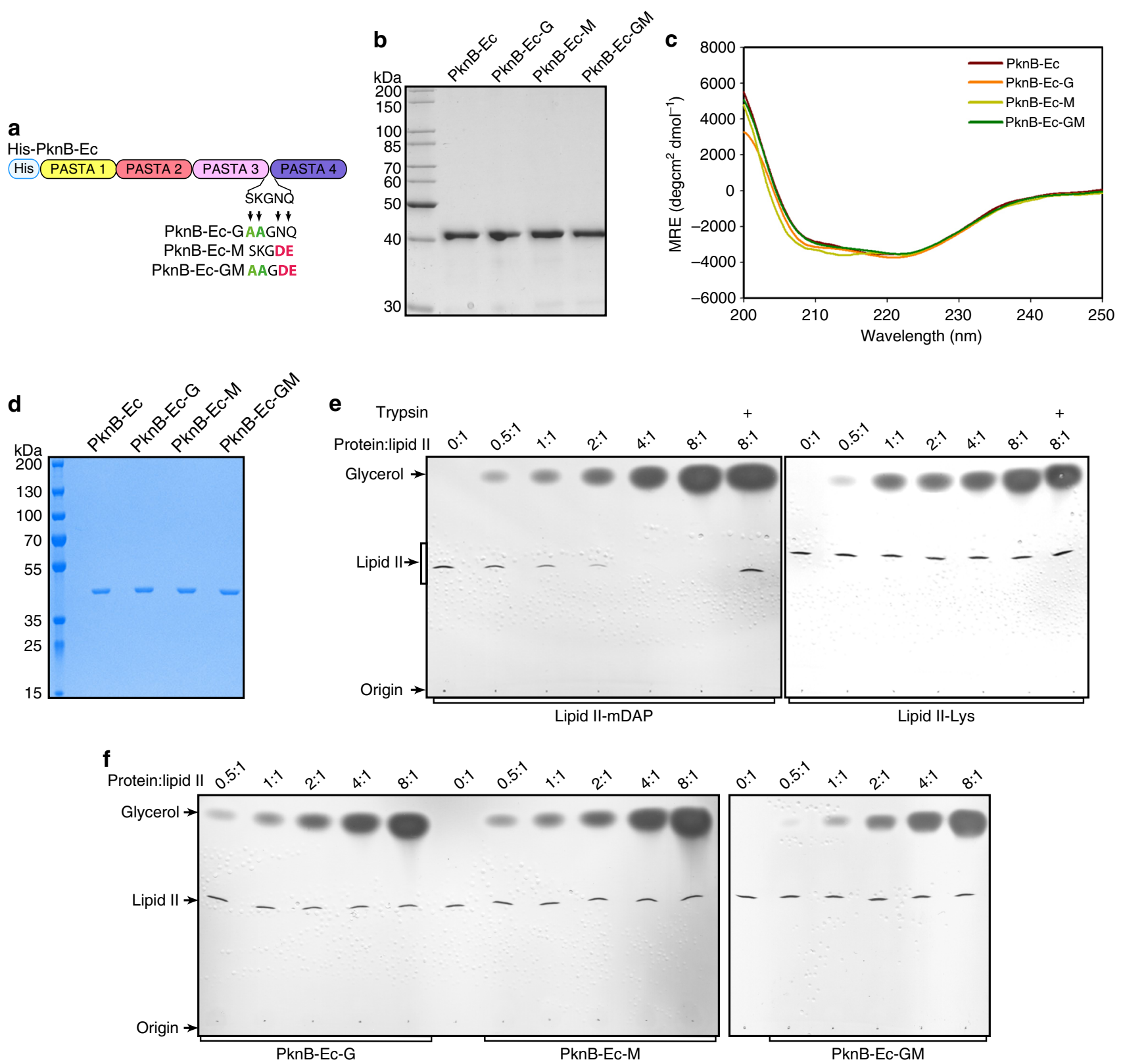

Fig. 4 Abrogation of ligand binding perturbs localization of PknB. a Schematic depicting hexa-His tagged extracytoplasmic region of PknB (PknB-Ec) and PknB-Ec mutants. $\mathbf{b}$ His-PknB wild type and mutants were purified as described in methods and $2 \mu \mathrm{g}$ of purified $P \mathrm{knB}_{\mathrm{B}} \mathrm{Ec}_{\mathrm{wt}} / \mathrm{mutant} \mathrm{proteins}$ were resolved on

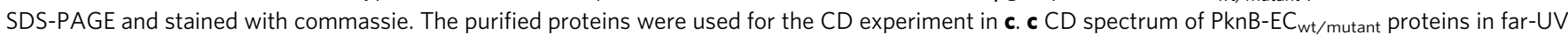
range (200-250 nm) CD data is depicted as MRE values (deg $\mathrm{cm}^{2} \mathrm{dmol}^{-1}$ ) in the $Y$-axis plotted against wavelength (nm) in $X$-axis. $\mathbf{d} 1 \mu \mathrm{g}$ each of His-

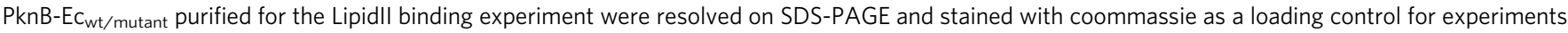
shown in e, f. e $2 \mathrm{nmol}$ of mDAP or Lys containing Lipidlls were incubated with increasing mole:mole ratio of His-PknB-Ec $\mathrm{c}_{w t}$ with respect to Lipidll. Addition of Trypsin to degrade His-PknB-Ec $c_{w t}$ in the reaction mixture is indicated. The samples were extracted with BuOH/PyrAc and resolved on TLC to analyze the presence of extractable Lipidll. Lipidll trapped in a stable complex with PknB Ec $c_{w t}$ resides in the water phase and free Lipidll is extracted and migrates to a defined position on the chromatogram. The intensity of the Lipidll bands relative to the control is shown. $\mathbf{f} 2 \mathrm{nmol}$ of mDAP containing Lipidll was incubated with increasing mole:mole ratio of His-PknB-Ec $\mathrm{wt}_{\mathrm{w}}$ mutant. The samples were extracted with $\mathrm{BuOH} / \mathrm{Pyr} \mathrm{Ac}$. Addition of Trypsin to degrade His-PknB-Ec $\mathrm{c}_{\mathrm{wt}}$ in the reaction mixture is indicated. The samples were extracted with organic solvent and resolved on TLC to analyze the presence of extractable Lipidll. Source data are provided as a Source Data file

targets of $\mathrm{PknB}$; and cluster 3 represents the proteins that are not the direct targets of $\mathrm{PknB}$, wherein phosphorylation does not alter significantly upon depletion or complementation with the wildtype PknB. (Fig. 7e; Supplementary Table 4c). Interestingly, we observed that both cluster 2 and cluster 3 proteins were hyperphosphorylated by the mutant PknB-GM, exemplifying both specific as well as promiscuous hyperphosphorylation (Fig. 7e; Supplementary Table 4c).
Hyperphosphorylation is a result of increased activity. To confirm that mutant is indeed hyper-phosphorylated in the activation loop (Fig. 6), we raised phosphospecific antibodies that are capable of recognizing phosphorylated T171 and T173 residues (Fig. 8a). The specificity and sensitivity of the antibodies were characterized (Supplementary Fig. 5). Consistent with mass spectrometry data, we observed $\sim 1.5$-fold increase in the normalized activation loop phosphorylation in the 
a

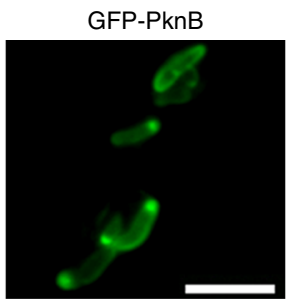

C

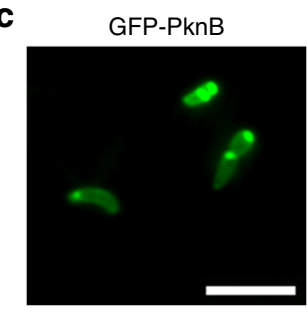

GFP-PknB + INH

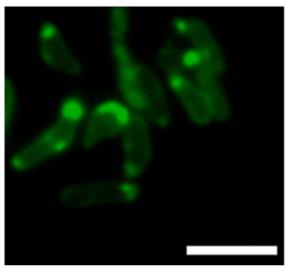

GFP-PknB + Nisin

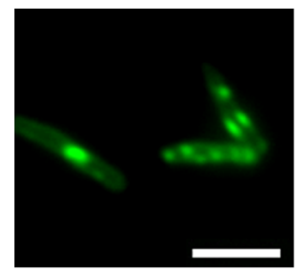

\section{e 4.5}

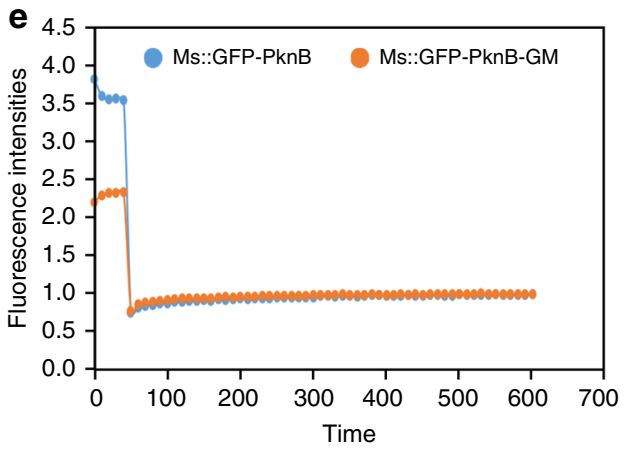

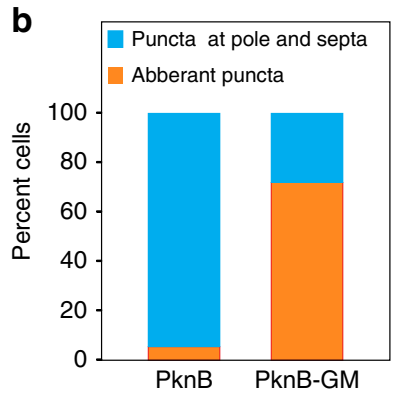

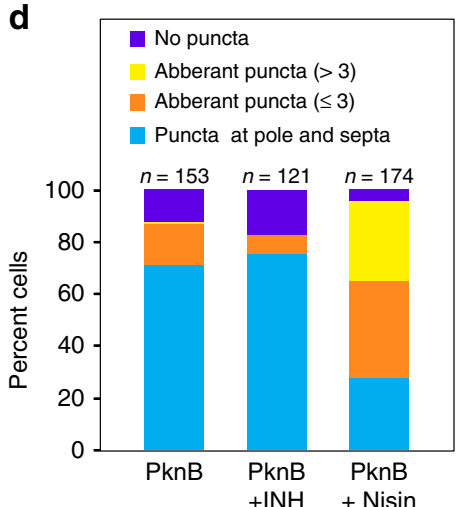

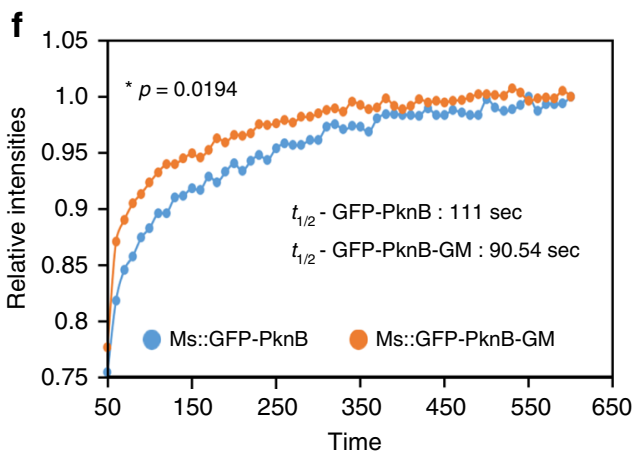

Fig. 5 Abrogation of ligand binding perturbs localization of PknB. a M. smegmatis $p k n B$ conditional mutant $\left(m c^{2} \Delta B\right.$ ) was electroporated with pNiT-GFP-PknB or pNit-GFP-PknB-GM constructs to generate $m c^{2} \Delta B:: G F P-B$ or $m c^{2} \Delta B:: G F P-B-G M$ strains. The strains were cultured in the presence of $50 \mathrm{ng}$ per ml ATc and $0.2 \mu \mathrm{M}$ IVN till $\mathrm{A}_{600}$ of $\sim 0.8$. The cultures were washed thrice with $\mathrm{PBST}_{80}$ to remove ATc and the cultures were grown for 6 hours in $7 \mathrm{H} 9$ media containing $1 \mu \mathrm{M}$ IVN. Florescence images were captured using $\times 100$ oil-immersion Zeiss Imager. M1 microscope. Scale bar- $5 \mu \mathrm{m}$. b $200 \mathrm{mc}{ }^{2} \Delta B:: g B$ or $m c^{2} \Delta B:: g B-G M$ cell from a were analyzed for the localization of GFP-PknB $B_{w t} / m u t a n t$. The aberrant puncta collectively represents cells without any distinct localization or showing puncta at regions other than poles and septa. $\mathbf{c} m c^{2} \Delta B:: g B$ strain cultured till $A_{600}$ of $\sim 0.8$ were washed and grown for 3 hours in $7 \mathrm{H} 9$ media containing $1 \mu \mathrm{M} \mathrm{IVN}$ in the presence or absence of $25 \mu \mathrm{g}$ per ml nisin or $250 \mathrm{ng}$ per ml INH. Scale bar- $5 \mu \mathrm{m}$. d Between 120 and 174 cells (as indicated) of $m c^{2} \Delta B:: g B$ cell from c were analyzed for the localization of GFP-PknB $B_{w t}$. The no puncta phenotype has been mentioned as a distinct feature from aberrant puncta in this case for better analysis of phenotype. Scale bar- $5 \mu \mathrm{m}$. e, $\mathbf{f}$ M. smegmatis $m c^{2} 155$ strain was electroporated with GFP-PknB or GFP-PknB-GM to generate $m c^{2}:: g B$ or $m c^{2}:: g B-G M$. Cultures of $m c^{2}:: g B$ or $m c^{2}:: g B-G M$ grown in the presence of $0.2 \mu M$ IVN were used for the FRAP analysis. $\mathbf{e}$ The graph represents the mean fluorescence intensity plotted as a function of time by normalizing the intensity at each time point $\left(I_{t}\right)$ to the first time point $\left(I_{0}, t=0 \mathrm{~s}\right)$ i.e., $I_{t} / I_{0}$. The values obtained were subtracted from the intensity at the time point of bleaching $\left(I_{b}\right)$. $f$ The time frames from 50 to $600 \mathrm{~s}$ are depicted, which highlight the difference in recovery times of GFP-PknB and GFP-PknB-GM. The $t_{1 / 2}$ values of recovery obtained are an indication of the time taken for half the maximal recovery after bleaching in three biologically different experiments. Statistical analysis was performed with the unpaired $t$-test using Graphpad software. Source data are provided as a Source Data file

ligand binding mutant PknB-GM (Fig. 8b). GarA has previously been demonstrated to be a robust in vitro substrate for $\mathrm{PknA}, \mathrm{PknB}$, and $\mathrm{PknG}^{36}$, hence we performed in vitro kinase assays with immunoprecipitated $3 \mathrm{~F}-\mathrm{PknB}$ and $3 \mathrm{~F}-\mathrm{PknB}-\mathrm{GM}$ using GarA as the substrate. It is apparent from the data that PknB-GM showed higher activity compared with the PknB (Fig. 8c, d), which could be a combinatorial effect of higher phosphorylation of loop as well as the juxtamembrane residues. Even though GarA is a robust in vitro substrate for $\mathrm{PknB}$, in in vivo it is majorly phosphorylated by PknG on T21 residue $^{36,37}$. In agreement with this we observed that phosphorylation of GarA on T21 is unperturbed by depletion of PknB as well as complementation (Fig. 7e). On the other hand, phosphorylation of TatA on T60 was found to be $\mathrm{PknB}$ dependent, which showed hyperphosphorylation upon complementation with PknB-GM (Fig. 7e). In an independent 
a

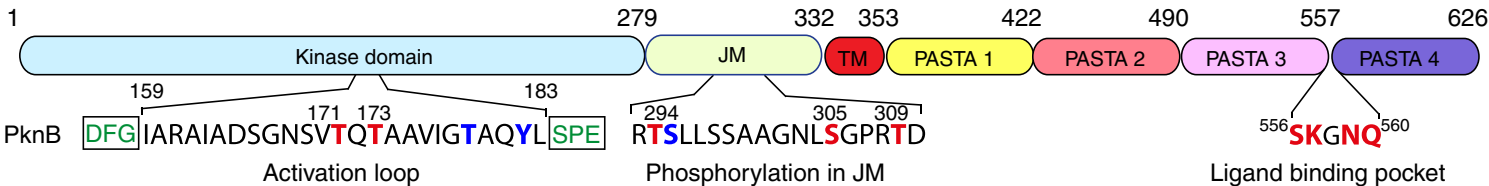

b

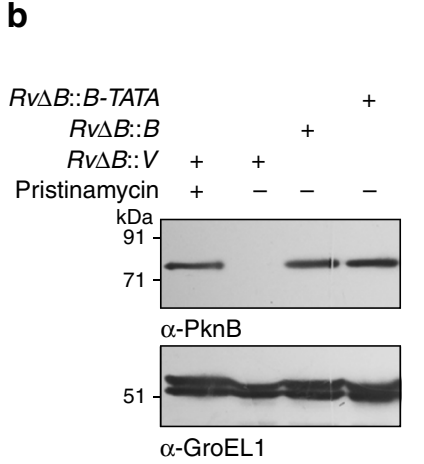

C

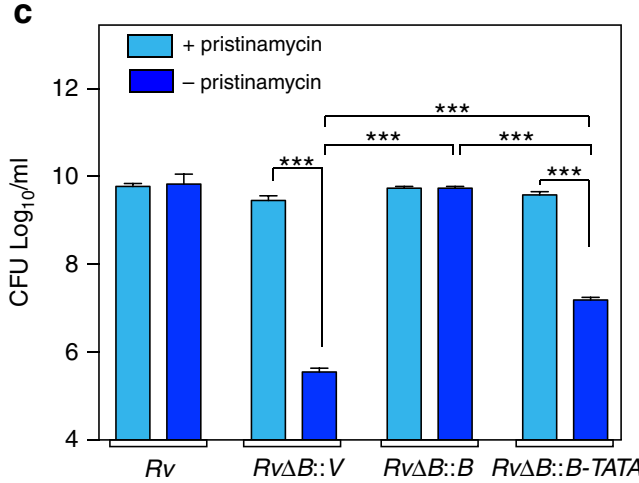

f

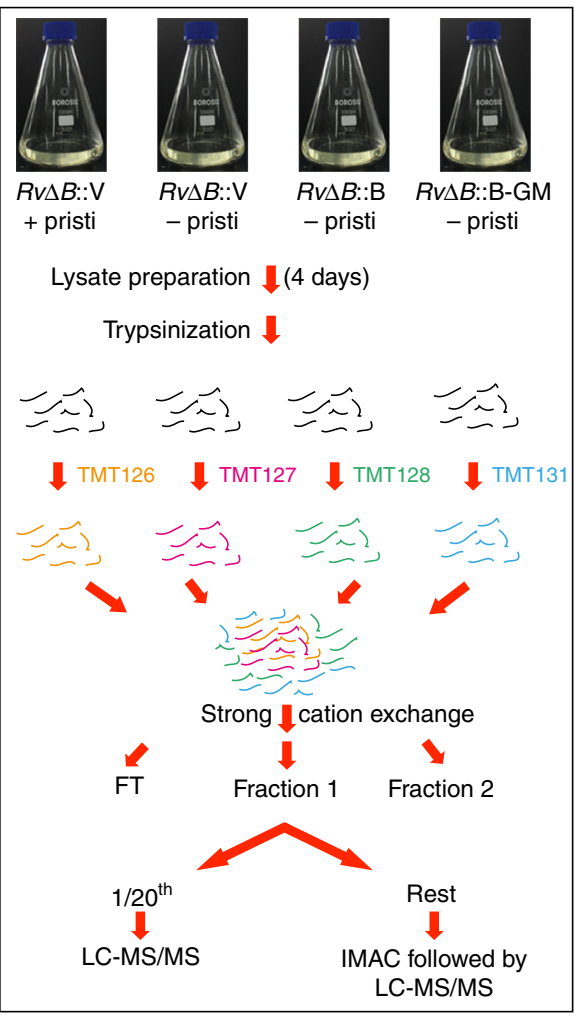

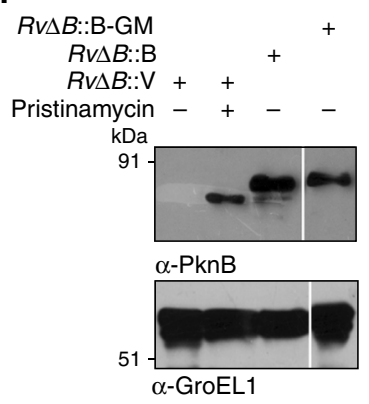

h d

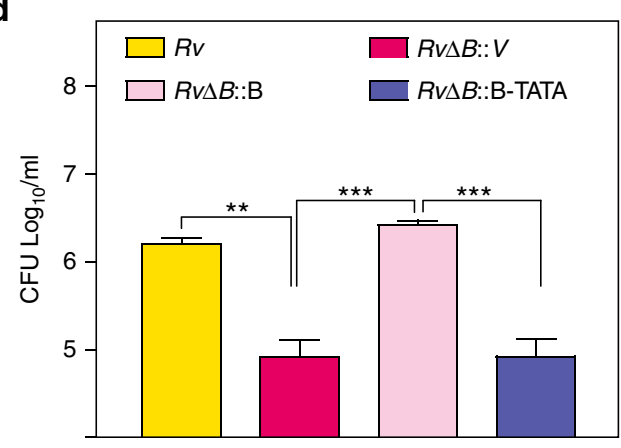

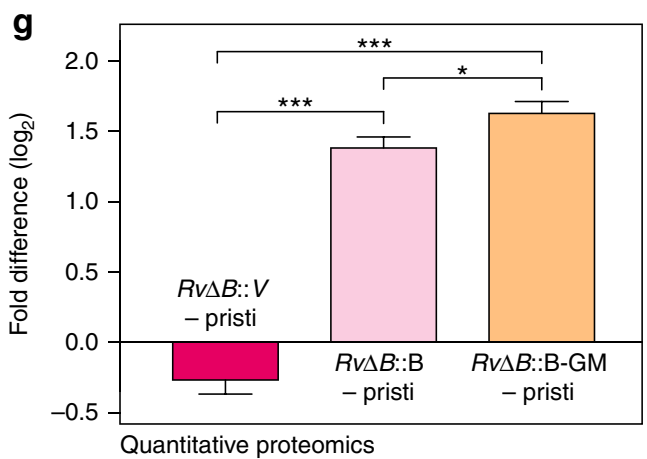

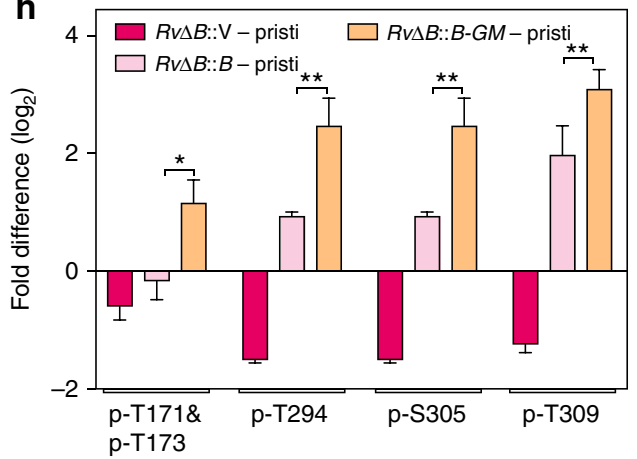

study, phosphorylation of TatA on T60 was shown to be dependent on PknA and $\mathrm{PknB}^{34}$.

We sought to validate the data by quantitating the peak area in an independent mass spec experiment for phosphopeptides corresponding to GarA(T21) and TatA(T60). Depletion of PknB in the absence of inducer and expression of PknB and PknB-GM was confirmed by western blots (Fig. 8e). In concurrence with the TMT data (Fig. 7), phosphopeptide corresponding to GarA-T21 showed similar peak area in $R v \Delta B$ \& $R v \Delta B:: B-G M$ samples, with slight decrease in $R v \Delta B:: B$ sample (Fig. 8f). On the other hand, phosphopeptide corresponding to TatA showed distinct hyperphosphorylation in $R v \Delta B:: B-G M$ compared with $R v \Delta B$ \& $R v \Delta B:: B$ (Fig. 8f). To further substantiate the data, we performed parallel reaction monitoring (PRM) to quantitate the TatA-T60 phosphopeptide (Supplementary Figs. 7 and 8g). Quantitation of TatA-T60 phosphopeptide with respect to the corresponding heavy peptide using PRM evidently demonstrated $\sim 2$-fold $(31.2$ fmoles) increase in its levels in $R v \Delta B:: B-G M$ sample compared with $R v \Delta B$ \& $R v \Delta B:: B$ (18.6 and 16.4 fmoles) samples. Collectively, these data demonstrate that the abrogation of ligand binding perturbs the normal regulatory circuits of $\mathrm{PknB}$, resulting in aberrant localization, hyperactivation of the kinase, and indiscriminate target-specific and promiscuous phosphorylation events, leading to eventual cell death (Fig. 8h)

\section{Discussion}

Since the domain structure of bacterial STPKs are similar to their eukaryotic counterparts $28,38,39$, the hypotheses with respect to 
Fig. 6 PknB-GM is hyper-phosphorylated in the activation loop. a Threonine and tyrosine residues that are be known to be phosphorylated are highlighted by red or blue color. The phosphorylations that are identified in the current study are shown in bold red. The ligand binding residues are also indicated. b $R v \Delta B$ strain was electroporated with integrating pST-CiT, pST-CiT-PknB, and pST-CiT-PknB-TATA to generate Rv $\Delta B:: V, R v \Delta B:: B$, and Rv $\Delta B:: B-T A T A$, respectively. The transformanats grown in the presence of pristinamycin were seeded at $A_{600}$ of $\sim 0.05$ and grown for 5 days in the presence or absence of pristinamycin. WCLs were resolved on SDS-PAGE and probed with $\alpha$-PknB and $\alpha$-GroEL1 antibodies. c Cultures of $R v$ and $R v \Delta B$ transformants were inoculated at $A_{600}$ of $\sim 0.05$ and grown in the presence or absence of pristinamycin for 6 days and CFUs were enumerated. Data are representative of one of the three biological experiments and each experiment was performed in triplicates. $\mathbf{d}$ Cultures of $R v$ and $R v \Delta B$ transformants grown till $\mathrm{A}_{600}$ of $\sim 0.6-1.0$ were washed thrice and used for infecting differentiated THP-1 cells (1:10 M.O.I). CFUs were enumerated at $72 \mathrm{~h}$ p.i. on plates containing pristinamycin. e Outline of the protocol used for TMT-based quantitative phosphoproteomics analysis. $\mathbf{f} R v B$ transformed with pNit-3F or pNit-3F-PknB or pNit-3F-PknBGM were grown in presence of pristinamycin or IVN for four days and WCLs prepared were probed with $\alpha$-PknB and $\alpha$-GroEL1 antibodies. $\mathbf{g}$ The absolute PknB levels in depleted and complemented strains were assessed by quantitative TMT-based proteomics approach. Each data point is represented by TMT intensities obtained from three technical replicates. Data represents mean $+\mathrm{SD}$. ${ }^{\star} p<0.05,{ }^{\star \star *} p<0.0005$. $\mathbf{h}$ The levels of phosphorylation of various residues in $\mathrm{PknB}$ activation loop and juxtamembrane regions were estimated from the TMT experiment. Phosphorylated peptides from three replicates normalized to their corresponding PknB protein intensities were considered for this analysis. The data is represented as log2 values and the raw data of the experiment is presented in Supplementary Table 1. Data represents mean + SD. Statistical analysis was performed with the unpaired $t$-test using Graphpad software. ${ }^{\star} p<0.05,{ }^{\star \star} p<0.005,{ }^{\star \star \star} p<0.0005$. Source data are provided as a Source Data file

their activation and regulation are influenced by the findings in eukaryotic kinases. There are two major mechanisms by which the activity of a protein kinase is regulated: (a) by modulating protein expression levels and (b) by limiting the levels of activity through phosphorylation and dephosphorylation of the activation loop residues. In case of $\mathrm{PknB}$, the following findings have cumulatively led to the formulation of the activation mechanism hypothesis presented in Fig. $1 \mathrm{~b}$ : (i) $\mathrm{PknB}_{\mathrm{Mtb}}$ is autophosphorylated in the activation loop and this phosphorylation is necessary and sufficient for its activity in vitro ${ }^{27}$, (Supplementary Fig. 4). (ii) $\mathrm{PknB}_{\mathrm{Mtb}}$ forms both back-to-back ${ }^{15}$ and front-to-front $\mathrm{PknB}^{14}$ dimers, and dimerization is a pre-requisite for activation loop phosphorylations ${ }^{13}$ (iii). The PASTA domain interacts with mDAP-containing muropeptides, and this domain is adequate for appropriate localization of the protein ${ }^{11}$. Interestingly, $\mathrm{PknB}$ protein expression levels are downregulated during dormancy ${ }^{9}$ and nutrient starvation ${ }^{10}$ and are upregulated during exponential growth $^{8}$ and resuscitation ${ }^{9}$, suggesting that $\mathrm{PknB}$ activity may also be regulated through the modulation of its expression pattern.

The PASTA domains across the bacterial kingdom share a highly conserved globular structure, although their sequences are diverse ${ }^{40}$. Various PASTA kinases have been demonstrated to harbor specific unique features. For example, a conserved arginine in PASTA3 has been shown to be a determining factor for ligand-binding in PrkC, the Bacillus subtilis $\mathrm{PknB}$ ortholog ${ }^{41}$. Similarly, three putative muropeptide binding sites in the hinge regions have been suggested to be the ligand binding pockets in StkP $\mathrm{P}^{42}$. Recently, a unique citrate-binding site ${ }^{19}$ and a hydrolase (LytB)-binding region ${ }^{16}$ have been defined in the terminal PASTA domains of PknB and StkP, respectively, thus implicating other roles for the domain in addition to muropeptide binding. In $S$. pneumoniae, the terminal PASTA domain of StkP is both necessary and sufficient for its function ${ }^{16}$. In $M t b$, we have previously reported that the terminal PASTA4 is absolutely essential for the function of $\mathrm{PknB}^{5}$. However, unlike in S. pneumoniae, we observe that the terminal PASTA4 is not sufficient; rather, appropriate length of the total PASTA domain is also vital (Fig. 1). The fact that PASTA4 plays an indispensible role is reinforced by the finding that $\mathrm{SK}$ and NQ residues in the PASTA3-4 linker region, serve as the putative ligand interacting residues (Fig. 2). In contrast to the tetra mutant that shows a drastic phenotype (Fig. 2), we observed varying levels of compromise in case of double mutants, with the data suggesting a greater role for mDAP-interacting residues compared with iGlninteracting residues (Fig. 3). Interestingly, both the double mutants as well the tetra mutant are similarly compromised in their ability to functionally complement $\mathrm{PknB}$ depletion ex-vivo, suggesting that $\mathrm{PknB}$ plays a very stringent role at the time of infection and under these circumstances even minor perturbations are not tolerated (Figs. 2 and 3).

Muropeptides are widely acknowledged as the ligands for the PASTA domains of $\mathrm{PknB}_{\mathrm{Mtb}}{ }^{11,12}$. However, some experimental observations suggest that they may not be the primary ligand: for example, in vitro binding affinity of muropeptides to purified $\mathrm{PknB}-\mathrm{Ec}$ is relatively weak, with micromolar concentrations of muropeptide being required for the binding $2,11,12$, and the growth inhibition due to overexpression of PknB-Ec could not be ameliorated upon the addition of muropeptides mixtures ${ }^{43}$. Optimal binding occurs only when the MurNAc sugar and the stempentapeptide (muramyl pentapeptide) are present in the ligand. The other possible source of PknB ligand is peptidoglycan precursors such as LipidII, also present in the periplasmic space. LipidII possesses all the signatures of the muropeptide ligand and is also spatio-temporally localized to the same niche as PknB. In line with this, $\mathrm{PknB}_{s a}$ was recently shown to bind very efficiently with LipidII molecules ${ }^{22}$. Our data also demonstrates efficient binding of PknB-Ec with mDAP-LipidII (Fig. 4). The weaker binding affinity compared to $\mathrm{PknB}_{s a}$ could be either due to the fact that we tested only the PknB-Ec, or could be due to the lack of additional modifications (such as amidation), known to be present in mDAP as well as iGlu residues in $M t b^{44}$. Importantly, all three PknB-Ec mutants failed to interact with the mDAPLipidII, clearly showing abrogation of ligand binding (Fig. 4).

The extracytoplasmic PASTA domain by itself has previously been shown to be sufficient for appropriate localization of $\mathrm{PknB}$, which suggested that the interaction of this domain with the ligand may dictate the localization ${ }^{11}$. The data presented in Figs. 4 and 5 of this study suggest that LipidII may be the primary intracellular ligand, as incubating the cells with sublethal doses of the drug "nisin" results in an aberrant distribution pattern of $\mathrm{PknB}$ in the cell. Previous studies have shown that the addition of purified intracellular PASTA domain to the culture also inhibits the growth of $M t b^{43}$. Considering this fact with the data in Fig. 5, it is evident that incubating the cells with purified extracytoplasmic PASTA domain or nisin results in sequestration of available LipidII, thus compromising the functionality of $\mathrm{PknB}$. The NMR structure of PASTA domain suggests that it is a linear domain. It is not clear as to how the muropeptide moiety in the LipidII interacts with the linear PASTA domain. It is possible that the interaction occur once the muropeptide is cleaved from the decaprenyl moiety. Alternatively, in the cellular context the PASTA domains may not be linear in structure. The regulation 
a

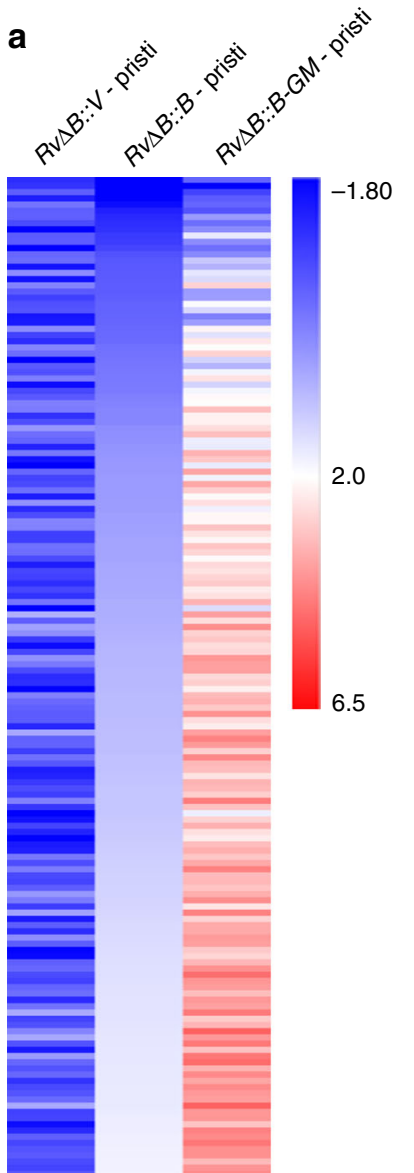

b

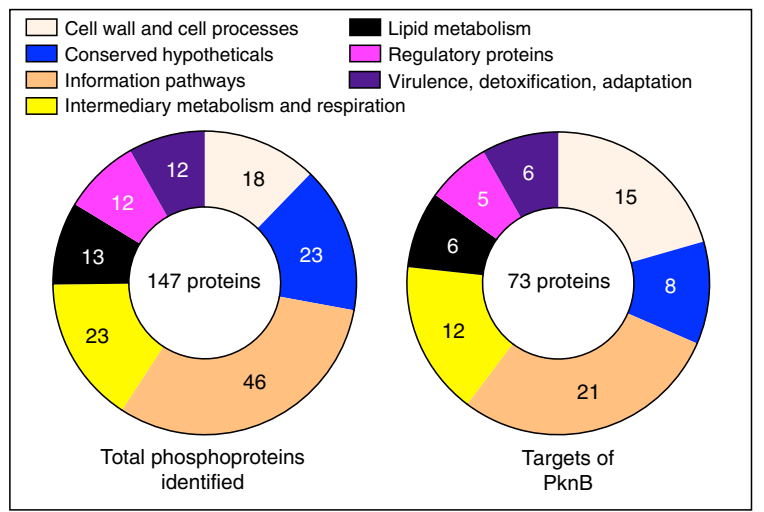

d

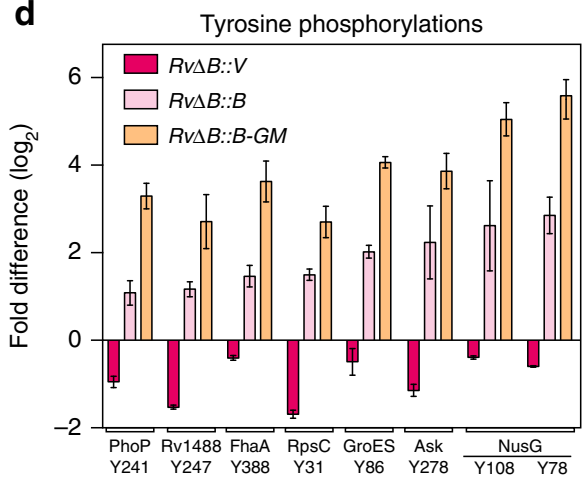

C

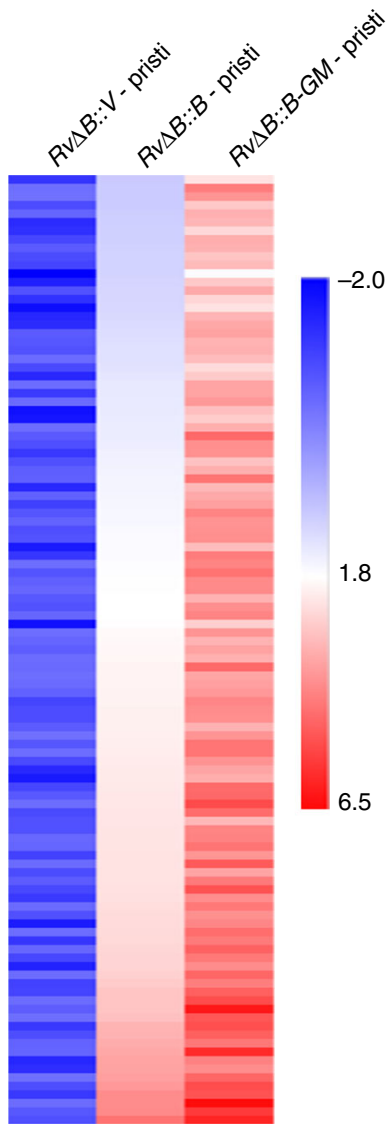

e Serine/threonine phosphorylations

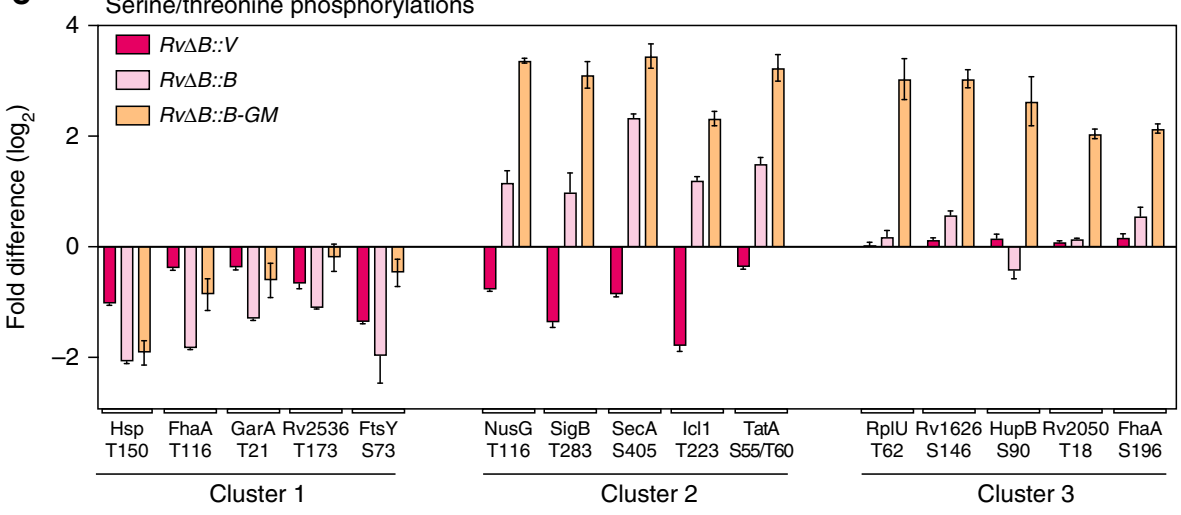

Fig. 7 Ligand binding mutation causes global hyperphosphorylation of specific and non-specific targets. a TMT intensities of phosphopetides in Rv $\Delta B$ pristinamycin (PknB depleted sample, labeled with TMT 127) or in Rv $\Delta B:: B$ (complemented with 3F-PknB, labeled with TMT 128) or Rv $\Delta B:: B-G M$ (complemented with 3F-PknB-GM, labeled with TMT131), with respect to Rv $\Delta B$ +pristinamycin as the reference comparator (control strain, labeled with TMT 126). The intensities of phosphopeptides in each case were normalized with respect to the corresponding absolute protein intensities and the values

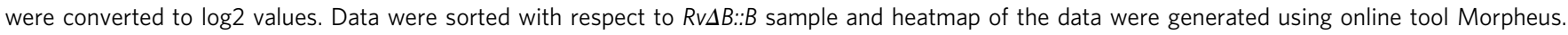
b The 257 phosphopeptides detected in TMT experiment belonged to 147 unique proteins, which were classified according to their functional category with reference to mycobrowser database. The phosphopeptides were categorized as probable PknB substrates if the TMT $\log _{2}$ phosphointensity upon depletion was $<-0.32$ and upon complementation was $>1$. 111 phosphopeptides were classified as probable PknB substrates, which belonged to 73 unique proteins. The 73 PknB targets were also functionally characterized according to mycobrowser database. c Normalized TMT intensities of all 111 phosphopeptides which are probable PknB targets were converted to $\log _{2}$ values and data were sorted with respect to Rv $\Delta B:: B$. Heatmap was generated using online tool Morpheus. d Normalized TMT intensities of 5 phosphopeptides each belonging to cluster 1, cluster 2, and cluster 3. e Normalized TMT intensities of PknBdependent tyrosine phosphorylations are represented. Source data are provided as a Source Data file 
a

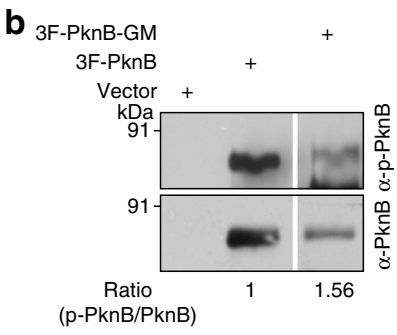

C

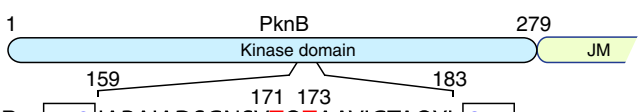

PknB DFGIARAIADSGNSVTQTAAVIGTAQYLSPE

Antigenic peptide [C]GNSVT(P)QT(P) AAV

$(\mathrm{p}-\mathrm{PknB} / \mathrm{PknB})$
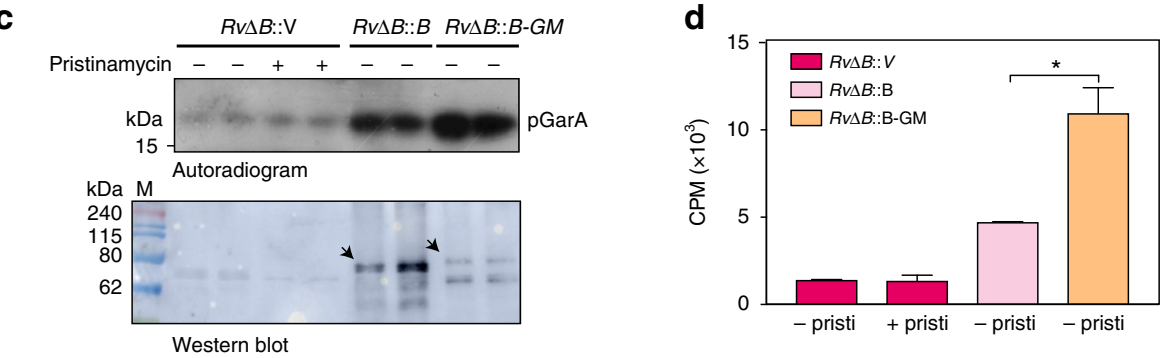

e
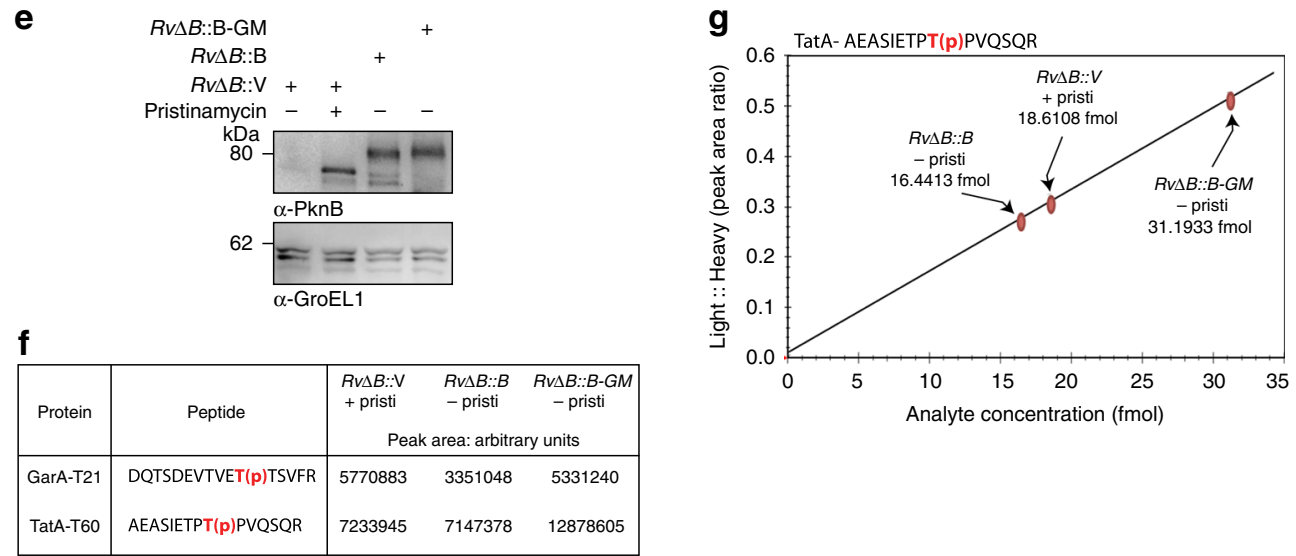

h

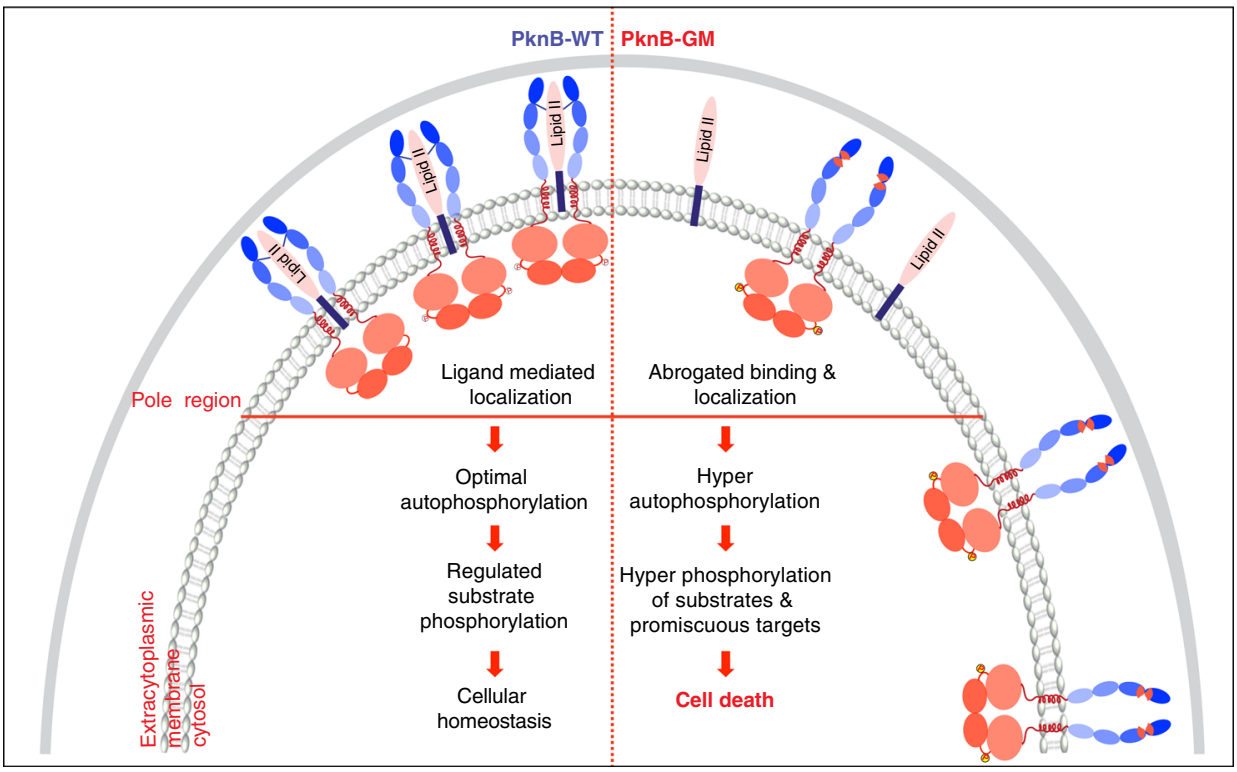

of the synthesis and continuous remodeling of PG is a fundamental process of the bacterial cell involving multiple proteins. $\mathrm{PknB}_{\mathrm{Mtb}}$ is a well-known modulator of multiple substrates involved in cell division and cell wall synthesis, phosphorylating MviN and FhaA proteins among others, a molecular event that is linked to reduced PG biosynthesis ${ }^{45,46}$. Thus, a feedback loop regulation mechanism intertwining $\mathrm{PknB}$ activity and peptidoglycan synthesis may exist. 
Fig. 8 Hyperphosphorylation is a result of increased activity of the PknB-GM mutant. a Schematic outline showing the activation loop region of PknB and the peptide sequence used for generating phospho-specific antibodies. b-d WCL were prepared from $R v \Delta B:: V$ grown in the presence and absence of pristinamycin; $R v \Delta B:: B$ and Rv $\Delta B:: B-G M$ grown in the absence of pristinamycin and in the presence of IVN for 4 days. $\mathbf{b}$ 3F-PknB or 3F-PknB-GM proteins were

immunoprecipitated from $1 \mathrm{mg}$ WCLs using FLAG-M2 beads and 1/10th of IP was probed with $\alpha$-PknB antibodies and $9 / 10^{\text {th }}$ of IP was loaded for $\alpha$-pPknB antibodies. The ratio of signal from total PknB and phospho-PknB was evaluated with the help of imageJ. $\mathbf{c}$ In vitro kinase assays samples were resolved on $15 \%$ SDS-PAGE, transferred to nitrocellulose membrane and autoradiographed. Upper part of the membrane corresponding to $40-240 \mathrm{kDa}$ was probed with $\alpha$-PknB antibody. $\mathbf{d}$ The bands corresponding to $\mathrm{p}-\mathrm{Gar} A(\sim 17 \mathrm{kDa})$ were excised from the membrane described in $\mathbf{c}$ and counts per minute (CPM) were determined (right panel). e $5 \mathrm{mg} W C L$ prepared from $R v \Delta B:: V$ + pristinamycin; $R v \Delta B:: B$-pristinamycin+IVN and Rv $\Delta B:: B$-GM -pristinamycin+IVN cultures grown for 4 days were digested with trypsin, and phosphoenriched. $\mathbf{f}$ We determined the peak area for GarA-T21 and TatA-T60 phosphopeptides using targeted proteomics. Table shows the total integrated area of GarA-T21 and TatA-T60 phosphopeptide in the samples. $\mathbf{g}$ Determination of absolute endogenous TatA-T60 phosphopeptide amounts in the samples by PRM. Graph showing absolute endogenous TatA-T60 light phosphopeptide concentration (fmoles) in phosphoenriched samples with respect to the standard curve generated by measuring peak area of different concentrations of heavy standard peptide (Supplementary Fig. 7). The horizontal axis represents Light: Heavy phosphopeptide integrated peak area ratios determined by skyline software. The vertical axis represents the absolute endogenous phosphopeptide TatA-T60 analyte concentration in fmoles. h Model depicting PknB regulation wherein Lipidll interacts with specific region of PASTA 3-4 linker region of PknB and defines its localization to polar/septal niches and regulates the activity to optimal levels and hence maintains cellular homeostasis and cell survival. Source data are provided as a Source Data file

If the ligand binding is required for the activation of $\mathrm{PknB}$, abrogation of binding should result in compromised loop phosphorylation. Contrary to this supposition, we observed significant hyperphosphorylation of activation loop and juxtamembrane regions in the PknB-GM mutant (Fig. 6). PrkC $\mathrm{bs}_{\mathrm{bs}}$ localizes to the division site and interacts and phosphorylates GpsB, which in turn regulates its activity by inhibiting its auto/transphosphorylations ${ }^{47}$. We hypothesize that upon binding of ligand, the kinase would be localized to the appropriate niche, whereupon a combination of other regulatory proteins/partners (including PstP) would ensure tight regulation of autophosphorylation levels, and by extension, kinase activity. In consonance, results (Fig. 8) showed that 3FPknB-GM to be a more active kinase compared with 3F-PknB. The mis-regulated kinase results in hyperphosphorylation events targeting proteins, which are both canonical and non-canonical substrates that may be leading to aberrant functionality, leading to eventual death (Fig. 7). PRM analysis showed $\sim 2$-fold increase in the phosphorylation of the substrate of TatA on T60 residue upon complementation with PknB-GM, thus validating the data (Fig. 8). There are few examples in literature wherein phosphomimetic mutant of PknB substrates such as InhA, KasB, PcaA, and CwlM have been shown to have significant impact on the catalytic function and/or survival defects ${ }^{46,48-51}$. Complementation with phosphomimetic mutant of KasB (T334D/T336D) results in loss of acid fastness character and also causes loss of virulence ${ }^{50}$. Phosphomimetic mutant of InhA (T266E) fails to rescue Msmeg/ $M t b$ inhA conditional mutant upon depletion ${ }^{48,49}$. PcaA-T168D/ T183D phosphomimetic mutant shows reduced bacterial survival and defective mycolic acid profile ${ }^{51}$. Recently, a double phosphomimetic mutant of a major substrate of PknB, CwlM $(\mathrm{T} 382 \mathrm{D}+\mathrm{T} 386 \mathrm{D})$ was shown to be defective in complementing the mutant strain ${ }^{46}$.

The hyperphosphorylation of the juxtamembrane domain could be linked to the hyperphosphorylation of the activation loop. Although the specific role of PknB juxtamembrane phosphorylation is unknown, it might be critical for transducing the ligand-mediated signal to the intracellular kinase domain, or for recruitment of regulatory interacting partners. Based on in vitro phosphorylation assays, Sassetti's group suggested that $\mathrm{PknB}$ and $\mathrm{PknH}$ are master regulators that are capable of phosphorylating multiple other kinases $^{6}$. It is possible that promiscuous hyperphosphorylation could be due to aberrant activation of other STPKs by mislocalized PknB. Even though, we did not find any other STPKs in our final phosphoproteome, we cannot negate this prospect. These aspects need further investigation. Future PknB interactome studies may provide possible insights into how PknB activation loop and juxtamembrane phosphorylations are regulated.

\section{Methods}

Generation of plasmid constructs. $p k n B$ was amplified from the genomic DNA of $M t b$ and the amplicon was cloned into NdeI-HindIII sites of pNit- $1^{52}$ and pNit-3F vectors to generate $\mathrm{pNit}-\mathrm{B}$ and $\mathrm{pNit}-3 \mathrm{~F}-\mathrm{B}$, respectively. $P k n B$ upto transmembrane region (NdeI-SapI) and PASTA-234 (SapI-HindIII) were PCR amplified and the digested amplicons were ligated with NdeI-HindIII digested pNit-3F to generate PknB-234. Similarly, $p k n B-12$ and PASTA-12 were ligated with pNit-3F to generate PknB-1212. PknB activation loop mutants and PASTA point mutants were generated by overlapping PCR. The codon optimized nucleotide sequence of superfolder $g f p$ was commercially synthesized from Genscript. $p k n B$ or $p k n B-G M$ were amplified using specific primers containing SapI and HindIII sites, and $s g f p$ was amplified from pUC57-sGFP using specific primers containing NdeI and SapI sites. The amplicons were digested and ligated with pNit-3F vector digested with NdeI-HindIII to generate pNit-GFP-PknB and pNit-GFP-PknB-GM. The extracytoplasmic domain of $p k n B_{\mathrm{wt} / \text { mutant }}$ was PCR amplified using pNit-3F$\mathrm{PknB}_{\mathrm{wt} / \mathrm{mutant}}$ as the template and the amplicons were digested with BamHIHindIII and cloned into corresponding sites in pET28a vector to generate pET$p k n B-E c$, pET-pknB-Ec-G, pET-pknB-Ec-M, and pET-pknB-Ec-GM. Oligonucleotides and strains used in the study are described in Supplementary Material.

Analysis of growth, isolation of lysates, and western blot. $p k n B$ conditional mutants, $m c^{2} \Delta B$ and $R v \Delta B$ (Rv-pptr-B $)^{5,17}$ were electroporated with pNit, pNit-3F or pST-CiT ${ }^{53}$ derived constructs (Supplementary Material). Transformants were grown in $7 \mathrm{H} 9$ media containing ADC (10\%) and pristinamycin $1 \mathrm{~A}(1.5 \mu \mathrm{g}$ per ml; Molcon Corp) till $\mathrm{A}_{600}$ reached $\sim 1.0$. To determine the ability of mutants to rescue growth, the cultures were washed thrice with equal volumes of $\mathrm{PBST}_{80}$ (1X PBS with $0.05 \%$ tween 80 ), diluted to $\mathrm{A}_{600}$ of $\sim 0.05$, and grown for 6 days in the presence or absence of $1.5 \mu \mathrm{g}$ per ml pristinamycin or $0.2 \mu \mathrm{M}$ isovaleronitrile (IVN). CFUs were enumerated after 6 days of growth. To evaluate the expression of 3X-FLAG tagged wild type and mutant $\mathrm{PknB}$ proteins, the transformants were grown in the absence of pristinamycin and presence of IVN for 5 days and probed with anti-PknB ( $\alpha$-PknB), anti-phosphoPknB ( $\alpha-p P k n B)$, or anti-GroEL1 ( $\alpha$ GroEL1) antibodies. $\alpha$-PknB and $\alpha$-GroEL1 antibodies were raised in rabbits and were used at 1:10,000 dilutions. The rabbit polyclonal phospho-specific antibodies were custom generated by PhosphoSolutions (Aurora, CO) using the antigenic peptide "[C]GNSVT(P)QT(P)AAV", a sequence derived from the PknB activation loop. For the $\alpha$-pPknB blot the membrane was blocked with $5 \%$ BSA followed by overnight incubation with $a$-pPknB $(1: 250$ dilution in $5 \%$ BSA $)$ at $4{ }^{\circ} \mathrm{C}$. Monoclonal $\alpha$-FLAG M2 (Sigma-F1804) antibody was used at 1:2500 dilution.

THP-1 infections. $R v$ or $R v \Delta B$ transformants grown up to $\mathrm{A}_{600}$ of $\sim 0.8$ in the presence of $1.5 \mu \mathrm{g}$ per $\mathrm{ml}$ pristinamycin were washed once with $\mathrm{PBST}_{80}$ and twice with PBS to remove Tween ${ }_{80}$ and pristinamycin and the cells were passed through $27 \mathrm{G}$ needle syringe to make them into single-cell suspension. Human monocytic THP1 cells (ATCC-TIB-202) were cultured in RPMI (Hyclone) supplemented with $10 \%$ FBS (Invitrogen). THP1 infections were performed with $5 \times 10^{5}$ cells seeded in 24-well plates that were differentiated with $10 \mathrm{nM}$ PMA for $24 \mathrm{~h}$. Differentiated cells were allowed to recover for $12 \mathrm{~h}$ prior infection with $\mathrm{Mtb}$ at 1:10 or 1:4 MOI${ }^{54}$ The extracellular bacteria were removed $4 \mathrm{~h}$ post infection by washing the cells thrice with sterile PBS, and this was considered as the zero time point. $0.2 \mu \mathrm{M}$ IVN was added in the media wherever $R v \Delta B:: B_{\text {wt/mutant }}$ transformants were used for the infection. Cells were lysed in $1 \mathrm{ml}$ of $0.1 \%$ triton-X100 at 0 and $72 \mathrm{~h}$ post infection and CFUs were enumerated on $7 \mathrm{H} 11$ plates containing $1.5 \mu \mathrm{g}$ per $\mathrm{ml}$ pristinamycin. 
Identification of the muropeptide binding site. The coordinates of the PASTA domains of mycobacterial PknB were obtained from the NMR structure $(2 \mathrm{KUI})^{18}$. The two monomeric PASTA3-4 domains were subjected to protein-protein docking using HADDOCK web server ${ }^{55,56}$. Surface exposed hydrophobic residues - Met586, Val593, Val604, and Ile619 - were treated as active residues. To investigate possible binding modes of muropeptide to the dimeric model of PASTA3-4 domains, muropeptide ( $N$-acetylglucosaminyl- $N$-acetylmuramyl-alaiso-Gln-meso-diaminopimelic acid-ala-ala; Molecular formula: $\mathrm{C}_{40} \mathrm{H}_{67} \mathrm{~N}_{9} \mathrm{O}_{21}$ ) was docked using AutoDock $4^{57}$. Coordinates of the muropeptide were generated using the Babel program of Open Babel package ${ }^{58}$ and PyMOL software was used for visualization (The PyMOL Molecular Graphics System, Version 1.5.0.4 Schrödinger, LLC). The ligand was allowed maximum possible flexibility with 27 rotatable bonds and Gasteiger atomic charges assigned to it. Coordinates of dimeric model of PASTA3-4 domains were kept rigid, and the ligand was allowed to explore the entire surface through the construction of a grid box, within the docking grid of $60 \times 45 \times 29 \AA^{3}$ with a grid spacing of 0.475 . The docking simulation involved 27,000 generations, and population size of 150 in each genetic algorithm (GA) run. Energy evaluations were carried out 2,500,000 times, and one best individual was chosen from each iteration of the 250 Lamarkian search GA runs. The rate of genetic mutations and cross-overs were set to 0.02 and 0.8 , respectively. The most stable binding mode of the ligand had a free energy of binding of $-4.29 \mathrm{kcal}$ per mol. The ligand-receptor interface on the PASTA dimer was defined as residues from each of the PASTA3-4 monomers, which had at least one atom within $5 \AA$ of any atom of the ligand.

\section{Purification and circular dichroism. E. coli BL21(DE3) (Stratagene) cells trans-} formed with the appropriate recombinant plasmid (pET28a-pknB-Ec, pET28a$p k n B-E c-G, p E T 28 \mathrm{a}-p k n B-\mathrm{Ec}-\mathrm{M}$, and pET28a-pknB-Ec-GM) were grown in $1 \mathrm{~L}$ LB-medium (50 $\mu \mathrm{g}$ per ml kanamycin) at $37^{\circ} \mathrm{C}$. At an $\mathrm{A}_{600}$ of 0.6 , IPTG was added at a final concentration of $1 \mathrm{mM}$ to induce expression of the recombinant proteins at $30^{\circ} \mathrm{C}$. The purification of hexa-His tagged protein was performed as described earlier ${ }^{48}$. Jasco J-815 spectopolarimeter was used for analyzing the ellipticity changes for each protein in far-UV $(195-250 \mathrm{~nm})$ wavelength range at $20^{\circ} \mathrm{C}$. The ellipticity changes were converted into MRE (Molar residual ellipticity) values and plotted against wavelength using SigmaPlot version 10.0.

In vitro LipidII interaction studies. In vitro LipidII binding assay was performed using purified $\mathrm{His}_{6}-\mathrm{PknB}-\mathrm{Ec}_{\mathrm{wt}}$ or PknB-Ec $\mathrm{c}_{\text {mutants. }} \mathrm{His}_{6}-\mathrm{PknB}_{-} \mathrm{Ec}_{\mathrm{wt}}$ or PknB$\mathrm{Ec}_{\text {mutants }}$ were incubated with $2 \mathrm{nmol}$ LipidII at molar ratios ranging from 0.5 to $8: 1$ (PknB:LipidII) in $50 \mathrm{mM} \mathrm{Tris/HCl} \mathrm{pH} \mathrm{7.0,} 5 \mathrm{mM} \mathrm{MgCl}_{2}, 60 \mathrm{~min}$ at $30^{\circ} \mathrm{C}^{22}$. The reaction mixture was extracted with an equal volume of butanol/pyridine acetate (2:1; vol:vol; $\mathrm{pH} 4.2)$ and analyzed by thin layer chromatography (TLC) ${ }^{22}$. For tryptic digestions, $25 \mu \mathrm{g}$ trypsin (Gibco) were added thereafter, and the mixture was incubated at $37^{\circ} \mathrm{C}$ for $60 \mathrm{~min}$.

Fluorescence microscopy. $m c^{2} \Delta B$ strain was electroporated with pNit-GFP-PknB or pNit-GFP-PknB-GM and the colonies exhibiting optimal GFP fluorescence were chosen. The cultures supplemented with $50 \mathrm{ng}$ per ml ATc and $0.2 \mu \mathrm{M}$ IVN (for inducing sGFP-PknB) were initiated at $\mathrm{A}_{600}$ of $\sim 0.025$. After $12 \mathrm{~h}$ of growth cultures were washed twice with $\mathrm{PBST}_{80}$ and supplemented with $7 \mathrm{H} 9$ containing 1 $\mathrm{mM}$ IVN. The cultures were withdrawn at 3 and $6 \mathrm{~h}$ post depletion of native $p k n B$ and fixed with $4 \%$ PFA for microscopic analysis. The fixed cultures $(5 \mathrm{ml})$ were washed twice with 1X PBS and re-suspended in $100 \mu \mathrm{l}$ PBS and $5 \mu \mathrm{l}$ of fixed cultures were placed under a coverslip on a glass slide and observed in epifluorescence microscope at $\times 100$ oil immersion using $488 \mathrm{~nm}$ excitation wavelength. For antibiotic treatment experiment, washed cultures supplemented with $7 \mathrm{H} 9$ media containing $1 \mathrm{mM} \mathrm{IVN}$ in the presence of $25 \mu \mathrm{g}$ per ml nisin or $250 \mathrm{ng}$ per $\mathrm{ml} \mathrm{INH}$ were grown for $3 \mathrm{~h}$ prior to fixation and microscopy.

FRAP measurements. Cultures of $m c^{2}-155$ electroporated with pNit-GFP-PknB or pNit-GFP-PknB-GM $\left(m c^{2}:: G F P-P k n B\right.$ or $\left.m c^{2}:: G F P-P k n B-G M\right)$ were seeded at $\mathrm{A}_{600}$ of $\sim 0.05$ and expression of GFP-PknB/PknB-GM was induced with $1 \mu \mathrm{M}$ of IVN for $16 \mathrm{~h}$ at $30^{\circ} \mathrm{C}$. For imaging, $100 \mu \mathrm{l}$ of culture spotted on a glass bottom dish and low-melting agarose ( $1 \%$ in $7 \mathrm{H} 9)$ was layered and allowed to settle and dry before FRAP measurements. For FRAP experiments, the bacilli containing the GFP signal were focused and one end of each bacteria (covering $30-50 \%$ area) was photo bleached using a $488 \mathrm{~nm}$ laser at $100 \%$ output power using a $3 \mathrm{i}$ vector system ( $3 \mathrm{i}$, USA) mounted on the Olympus IX83 inverted microscope, controlled using the Slidebook 6.0 software. The fluorescence recovery was measured by time-lapse imaging in the GFP channel ( $488 \mathrm{~nm}$ excitation through a SpectraX light engine and emission filter of $510 / 20 \mathrm{~nm}$ ), using the $\times 601.35$ NA plan-apochromat lens. The images were acquired using a Cascade II EM-CCD camera (Photometrics, USA) with EM gain for a total duration of 10 min with $10 \mathrm{~s}$ interval between two successive frames ${ }^{59}$. To plot the fluorescence recovery kinetics, the intensity normalized at each time point $\left(I_{\mathrm{t}}\right)$ to the first time point $\left(I_{0}, t=0 \mathrm{~s}\right)$. The values obtained were subtracted from the intensity at the time point of bleaching $\left(I_{\mathrm{b}}\right)[=$ $\left.\left(I_{t} / I_{0}\right)-I_{\mathrm{b}}\right]$, allowing the recovery to be recorded with respect to $I_{\mathrm{b}}$. The polar regions of $\sim 120$ cells for each sample were photo-bleached at the fifth frame (50 s) and the fluorescence recovery was monitored up to $600 \mathrm{~s}$ from at least three biologically independent experiments. The mean relative intensity obtained was plotted as a function of time (in seconds) to calculate the rate of recovery $\left(t_{1 / 2}\right.$, the time taken to attain half of the maximum intensity) in the bleached areas of each bacterium and plotted using the Non-linear fit equation in Prism 6.0.

Immunoprecipitation and in vitro kinase assays. $R v \Delta B$ strains electroporated with pNit-3F-PknB $\mathrm{WT}_{\mathrm{WTM}}$ were inoculated at $\mathrm{A}_{600}$ of $\sim 0.05$ and were grown in the absence of pristinamycin and presence of $0.2 \mu \mathrm{M}$ IVN for $72 \mathrm{~h}$. Cultures were centrifuged at $4000 \mathrm{rpm}$ for $5 \mathrm{~min}$ and the pellets were resuspended in PBSG (PBS containing 5\% glycerol) at 1:3 (pellet weight in g:PBSG volume in ml) ratio. Cells were lysed using $0.1 \mathrm{~mm}$ zirconium beads (Biospec) for $101 \mathrm{~min}$ cycles with $1 \mathrm{~min}$ intervals on ice using Biospec minibeadbeater. The lysates were clarified at 13,000 rpm at $4{ }^{\circ} \mathrm{C}$ and the concentration of supernatant whole-cell lysates (WCLs) were estimated. $1 \mathrm{mg}$ of WCL was used for FLAG immunoprecipitation (IP) using FLAG-M2 beads and the $3 \mathrm{~F}-\mathrm{PknB}$ or $3 \mathrm{~F}-\mathrm{PknB}$-GM were eluted with $0.1 \mathrm{M}$ glycine $(\mathrm{pH} 2.2)$ and the eluate was neutralized by addition of $1 / 10$ th vol $1 \mathrm{M}$ Tris-HCl ( $\mathrm{pH}$ 8.0). The IPed proteins were resolved on SDS-PAGE and subjected to western blotting. The western blots were probed with 1:10,000 \& 1:250 dilution of $\alpha$-PknB and $\alpha$-pPknB antibodies. The ratio of phospho-PknB band to that of PknB was evaluated using image $\mathrm{J}^{60}$. The in vitro kinase assays were performed in $30 \mu \mathrm{l}$ reaction volume containing $25 \mathrm{mM}$ HEPES-NaOH, $\mathrm{pH}$ 7.4, $20 \mathrm{mM}$ magnesium acetate, $20 \mathrm{mM} \mathrm{MnCl}_{2}, 1 \mathrm{mM}$ DTT, $100 \mu \mathrm{M}$ ATP, $10 \mu \mathrm{Ci}$ of [ $\left.\gamma^{32} \mathrm{P}\right] \mathrm{ATP}$, and $2 \mu \mathrm{g}$ of $\mathrm{GarA}$ and $10 \mu \mathrm{l}$ eluate from IP for $30 \mathrm{~min}$ at $30^{\circ} \mathrm{C}$. The reactions were stopped by adding $15 \mu \mathrm{l}$ of $6 \mathrm{X}$-SDS sample buffer followed by heating at $95^{\circ} \mathrm{C}$ for $5 \mathrm{~min}$ Reactions were resolved on $15 \%$ SDS-PAGE, transferred to nitrocellulose membrane and autoradiographed. The bands corresponding to pGarA were excised, incubated overnight in the scintillation cocktail (spectrochem) and counts per minute (CPM) were determined using Perkin Elmer microbeta TriLux 1450 LSC \& Luminescence counter.

TMT labeling and MS/MS analysis. Cultures pellets were resuspended in SDS lysis buffer (2\% SDS, $50 \mathrm{mM}$ Triethylammonium bicarbonate buffer (TEAB; sigma), PhosSTOP tablets (Roche)in PBSG (PBS containing 5\% glycerol) at 1:3 (pellet weight in g:buffer) ratio. Samples were heated at $95^{\circ} \mathrm{C}$ for $20 \mathrm{~min}$ followed by 10 cycles of beadbeating. The lysates were clarified and the concentration of supernatant WCLs were estimated with the help of BCA reagent (Pierce). WCL $(250 \mu \mathrm{g})$ from each samples shown in Fig. 6e were reduced using $10 \mathrm{mM}$ tris (2-carboxyethyl) phosphine at $55^{\circ} \mathrm{C}$ for $1 \mathrm{~h}$ and alkylated using $10 \mathrm{mM}$ iodoacetamide for $30 \mathrm{~min}$ at $25^{\circ} \mathrm{C}$. Samples were acetone precipitated and the pellet was resuspended in $100 \mu \mathrm{l}$ of $100 \mathrm{mM} \mathrm{TEAB}$ and digested with $6 \mu \mathrm{g}$ Trypsin (Promega) for $16 \mathrm{~h}$ at $37^{\circ} \mathrm{C}$. TMT labeling (Thermo Fisher Scientific) was performed as per manufacturer's instructions. Peptides from $R v \Delta B:: V+$ pristinamycin; $R v \Delta B:: V$ - pristinamycin; $R v \Delta B:: B$ pristinamycin + IVN; and $R v \Delta B:: B-G M$ - pristinamycin + IVN were labeled with $126,127,128$, and 131 reporter ions respectively. Labeled samples were pooled, dried, and Strong Cation Exchange chromatography (SCX) was performed using two salt gradients, 100 and $350 \mathrm{mM} \mathrm{KCl}^{61} .1 / 20$ th of each fraction was secured for the total proteome analysis. Rest of the sample was enriched for the phosphopeptide using IMAC beads as described earlier ${ }^{62}$

The desalted samples for the total proteome analysis as well as the enriched peptide samples for phosphoproteomics analysis were reconstituted in Buffer A (95\% Water, 5\% Acetonitrile, 0.1\% Formic acid). All experiments were performed using EASY-nLC system (Thermo Fisher Scientific) coupled to LTQ OrbitrapVelos mass spectrometer (Thermo Fisher Scientific) equipped with nanoelectrospray ion source. A $10-\mathrm{cm}$ PicoFrit Self-Pack microcapillary column (New Objective) was used to resolve the peptide mixture and the peptides were eluted at a flow rate of $300 \mathrm{nl}$ per min for $120 \mathrm{~min}$. The acetonitrile (containing $0.1 \%$ formic acid) gradient used for the run was $0-40 \%$ for $70 \mathrm{~min}, 40-80 \%$ for 10 min, $80 \%$ for $10 \mathrm{~min}, 80-0 \%$ for $5 \mathrm{~min}$, and $0 \%$ for last $25 \mathrm{~min}$ LTQ orbitrap was used for the full MS scan. The peptides were dissociated with both HCD and CID for better MS/MS spectra. The collision energy induced dissociation of $\mathrm{X}$ ion precursors was performed at 35 for CID and 40 for HCD. Both MS and MS/MS data were acquired using scan range of $20-2000 \mathrm{M} / \mathrm{Z}$ ratios. The dynamic exclusion was set at 500 for both ion trap (CID) and FTMS (HCD) and the resolution was set at 7500 . Spectra obtained were queried against $M t b$ H37Rv database (refseq database 85, release date 11 January 2018). Proteome Discoverer 1.3 was used as the search algorithm with oxidation of methionine and carbamidomethylation of cysteine as static modification. Phosphorylation of serine, threonine, and tyrosine was used as a dynamic modification. TMT 6 plex modification of peptide $\mathrm{N}$ termini and lysine residues were set as the fixed modification. The TMT ratios were calculated with TMT126 ( $R v \Delta B:: V$ + pristinamycin sample) as the reference comparator. All PSMs were identified at $1 \%$ false discovery rate (FDR). Mass tolerance for precursor ions and fragment ions were set at $10 \mathrm{ppm}$ and $0.1 \mathrm{Da}$ respectively. Mass-spectrometry analysis was performed to obtain a list of intensities of various proteins in proteome and phospho-enriched proteome. The phosphorylation sites in the protein for the phosphopeptides were identified with the help of in-house scripts tool for the proteomic analysis (http://protocols. ibioinformatics.org/InHouse/). A localization probability cutoff of $75 \%$ was defined in the in-house scripts proteomic analysis tool prior to $\mathrm{p}$-site analysis. The $\mathrm{p}$-sites hence identified were further analyzed for pRS score and PEP values. The p-sites with pRS score $>50$ and PEP $<0.05$ were only considered for further analysis. The 
common proteins represented in the total proteome and phosphoproteome were analyzed using venn diagram generating tool venny ${ }^{63}$. The phosphointensity ratio of each phosphopeptide was normalized against the whole-protein intensity ratio of the corresponding protein. The normalized phosphointensity from three replicates was averaged and the average phosphointensity ratios were converted into $\log _{2}$ values. Heatmap was generated using online tool Morpheus (https://software. broadinstitute.org/morpheus/). The functional characterization of individual phosphoproteins identified in the study was done using mycobrowser database. The TMT-phosphoproteomics data has been submitted to the ProteomeXchange Consortium (http://proteomecentral.proteomexchange.org) ${ }^{64}$ and can be accessed using data set identifier PXD012180 via the PRIDE partner repository.

Targeted proteomics. Synthetic isotopically labeled (SIL) peptides (Maxi SpikeTides QL_AAA-peptides) with C-terminus ${ }^{15} \mathrm{~N}$ and ${ }^{13} \mathrm{C}$-labeled arginine were purchased from JPT Peptide Technologies GmbH (Berlin, Germany). The synthetic peptides were resuspended in $0.1 \%$ Formic acid at a final concentration of $1.7 \mu \mathrm{g}$ per $\mu \mathrm{l}$ and working concentrations of $0.6,6,60$, and $600 \mathrm{fmol}$ per $\mu \mathrm{l}$ were prepared. All the samples were analyzed using EASY-nLC 1000 system (Thermo Fisher Scientific) coupled to Thermo Fisher-Orbitrap Q- Exactive mass spectrometer (Thermo Scientific, Germany) equipped with nanoelectrospray ion source. Samples were loaded with buffer A and eluted with a 0-40\% split gradient of buffer B ( $95 \%$ acetonitrile, $0.1 \%$ formic acid) at a flow rate of $300 \mathrm{nl}$ per min for $30 \mathrm{~min}$ using 25 $\mathrm{cm}$ PicoFrit column. The acquisition method had a non-scheduled parallel reaction monitoring (PRM) event targeting the doubly charged precursor ion of the SIL peptides. The PRM event was performed with an orbitrap resolution of 17500 (at $\mathrm{m} / \mathrm{z} 200$ ), a target AGC value of 1e6, and maximum fill times of $100 \mathrm{~ms}$. Fragmentation was acquired with a normalized collision energy of $27 \mathrm{eV}$ and MS/MS scan range of $m / z 100-1500$ was used for mass determination. PRM data analysis was performed using skyline-Daily software version 4.1.1.18179 (https://skyline. $\mathrm{ms} /$ project/home/begin.view?). For reproducibility and precision of the PRM method, calibration graph was generated in skyline against different SIL peptide concentrations and most confident and intense transitions of the SIL peptide were used for quantification purpose. A fixed amount of SIL peptide ( $60 \mathrm{fmol}$ ) was spiked in each phospho-enriched sample $(2 \mu \mathrm{g})$ for absolute concentration determination of endogenous phosphopeptide. The skyline file for data analysis has been attached as Supplementary Data 4, 5 and 6.

\section{Data availability}

The TMT-phosphoproteomics raw data associated with Figs. 6 and 7 has been submitted to the ProteomeXchange Consortium (http://proteomecentral.proteomexchange.org) and can be accessed using data set identifier PXD012180 via the PRIDE partner repository. The Skyline files for the PRM data analysis (Fig. 8) are submitted as Supplementary Data 4-6. Images of unprocessed western blots, coomassie stained gels, TLC images, and autoradiograms used in the study are provided in Supplementary Fig. 8. The source data underlying Figs. 1a, f, 2d-e, 3c-g, 4c, 5b-d, 6c, d, 7d, e, and $8 \mathrm{~d}$ are provided as a Source Data file. The source data underlying Fig. 6 are provided in Supplementary Table 1.

Received: 5 June 2018 Accepted: 28 February 2019

Published online: 15 March 2019

\section{References}

1. Jones, G. \& Dyson, P. Evolution of transmembrane protein kinases implicated in coordinating remodeling of gram-positive peptidoglycan: inside versus outside. J. Bacteriol. 188, 7470-7476 (2006).

2. Manuse, S., Fleurie, A., Zucchini, L., Lesterlin, C. \& Grangeasse, C. Role of eukaryotic-like serine/threonine kinases in bacterial cell division and morphogenesis. FEMS Microbiol. Rev. 40, 41-56 (2016).

3. Sassetti, C. M., Boyd, D. H. \& Rubin, E. J. Comprehensive identification of conditionally essential genes in mycobacteria. Proc. Natl Acad. Sci. USA 98, 12712-12717 (2001).

4. Fernandez, P. et al. The Ser/Thr protein kinase PknB is essential for sustaining mycobacterial growth. J. Bacteriol. 188, 7778-7784 (2006).

5. Chawla, Y. et al. Protein kinase B (PknB) of Mycobacterium tuberculosis is essential for growth of the pathogen in vitro as well as for survival within the host. J. Biol. Chem. 289, 13858-13875 (2014).

6. Baer, C. E., Lavarone, A. T., Alber, T. \& Sassetti, C. M. Biochemical and spatial coincidence in the provisional Ser/Thr protein kinase interaction network of Mycobacterium tuberculosis. J. Biol. Chem. 289, 20422-20433 (2014).

7. Richard-Greenblatt, M. \& Av-Gay, Y. Epigenetic phosphorylation control of Mycobacterium tuberculosis infection and persistence. Microbiol. Spectr. 5, 10.1128/microbiolspec.TBTB2-0005-2015 (2017).

8. Kang, C. M. et al. The Mycobacterium tuberculosis serine/threonine kinases PknA and PknB: substrate identification and regulation of cell shape. Genes Dev. 19, 1692-1704 (2005).
9. Ortega, C. et al. Mycobacterium tuberculosis Ser/Thr protein kinase B mediates an oxygen-dependent replication switch. PLoS Biol. 12, e1001746 (2014).

10. Betts, J. C., Lukey, P. T., Robb, L. C., McAdam, R. A. \& Duncan, K. Evaluation of a nutrient starvation model of Mycobacterium tuberculosis persistence by gene and protein expression profiling. Mol. Microbiol. 43, 717-731 (2002)

11. Mir, M. et al. The extracytoplasmic domain of the Mycobacterium tuberculosis Ser/Thr kinase PknB binds specific muropeptides and is required for PknB localization. PLoS Pathog. 7, e1002182 (2011).

12. Wang, Q. et al. A comprehensive study of the interaction between peptidoglycan fragments and the extracellular domain of Mycobacterium tuberculosis Ser/Thr kinase PknB. Chembiochem 18, 2094-2098 (2017).

13. Lombana, T. N. et al. Allosteric activation mechanism of the Mycobacterium tuberculosis receptor Ser/Thr protein kinase, PknB. Structure 18, 1667-1677 (2010).

14. Mieczkowski, C., Iavarone, A. T. \& Alber, T. Auto-activation mechanism of the Mycobacterium tuberculosis PknB receptor Ser/Thr kinase. EMBO J. 27, 3186-3197 (2008)

15. Wehenkel, A. et al. The structure of PknB in complex with mitoxantrone, an ATP-competitive inhibitor, suggests a mode of protein kinase regulation in mycobacteria. FEBS Lett. 580, 3018-3022 (2006).

16. Zucchini, L. et al. PASTA repeats of the protein kinase StkP interconnect cell constriction and separation of Streptococcus pneumoniae. Nat. Microbiol. 3, 197-209 (2018).

17. Forti, F., Crosta, A. \& Ghisotti, D. Pristinamycin-inducible gene regulation in mycobacteria. J. Biotechnol. 140, 270-277 (2009).

18. Barthe, P., Mukamolova, G. V., Roumestand, C. \& Cohen-Gonsaud, M. The structure of PknB extracellular PASTA domain from mycobacterium tuberculosis suggests a ligand-dependent kinase activation. Structure 18, 606-615 (2010)

19. Prigozhin, D. M. et al. Structural and genetic analyses of the Mycobacterium tuberculosis Protein Kinase B sensor domain identify a potential ligandbinding site. J. Biol. Chem. 291, 22961-22969 (2016).

20. Crick, D. C., Mahapatra, S. \& Brennan, P. J. Biosynthesis of the arabinogalactan-peptidoglycan complex of Mycobacterium tuberculosis. Glycobiology 11, 107R-118R (2001).

21. Mahapatra, S. et al. Mycobacterial lipid II is composed of a complex mixture of modified muramyl and peptide moieties linked to decaprenyl phosphate. J. Bacteriol. 187, 2747-2757 (2005).

22. Hardt, P. et al. The cell wall precursor lipid II acts as a molecular signal for the Ser/Thr kinase PknB of Staphylococcus aureus. Int. J. Med. Microbiol. 307, $1-10$ (2017)

23. Jani, C. et al. Regulation of polar peptidoglycan biosynthesis by Wag31 phosphorylation in Mycobacteria. BMC Microbiol. 10, 327 (2010).

24. Breukink, E. et al. Use of the cell wall precursor lipid II by a pore-forming peptide antibiotic. Science 286, 2361-2364 (1999).

25. Wiedemann, I. et al. Specific binding of nisin to the peptidoglycan precursor lipid II combines pore formation and inhibition of cell wall biosynthesis for potent antibiotic activity. J. Biol. Chem. 276, 1772-1779 (2001).

26. Hsu, S. T. et al. The nisin-lipid II complex reveals a pyrophosphate cage that provides a blueprint for novel antibiotics. Nat. Struct. Mol. Biol. 11, 963-967 (2004).

27. Duran, R. et al. Conserved autophosphorylation pattern in activation loops and juxtamembrane regions of Mycobacterium tuberculosis Ser/Thr protein kinases. Biochem. Biophys. Res. Commun. 333, 858-867 (2005).

28. Young, T. A., Delagoutte, B., Endrizzi, J. A., Falick, A. M. \& Alber, T. Structure of Mycobacterium tuberculosis PknB supports a universal activation mechanism for Ser/Thr protein kinases. Nat. Struct. Biol. 10, 168-174 (2003).

29. Prisic, S. et al. Extensive phosphorylation with overlapping specificity by Mycobacterium tuberculosis serine/threonine protein kinases. Proc. Natl Acad. Sci. USA 107, 7521-7526 (2010).

30. Fortuin, S. et al. Phosphoproteomics analysis of a clinical Mycobacterium tuberculosis Beijing isolate: expanding the mycobacterial phosphoproteome catalog. Front. Microbiol. 6, 6 (2015).

31. Verma, R. et al. Quantitative proteomic and phosphoproteomic analysis of H37Ra and H37Rv strains of Mycobacterium tuberculosis. J. Proteome Res. 16, $1632-1645$ (2017)

32. Sajid, A. et al. Interaction of Mycobacterium tuberculosis elongation factor $\mathrm{Tu}$ with GTP is regulated by phosphorylation. J. Bacteriol. 193, 5347-5358 (2011).

33. Gupta, M. et al. HupB, a nucleoid-associated protein of Mycobacterium tuberculosis, is modified by serine/threonine protein kinases in vivo. $J$. Bacteriol. 196, 2646-2657 (2014).

34. Carette, X. et al. Multisystem Analysis of Mycobacterium tuberculosis Reveals Kinase-Dependent Remodeling of the Pathogen-Environment Interface. MBio 9, e02333-17 (2018)

35. Kusebauch, U. et al. Mycobacterium tuberculosis supports protein tyrosine phosphorylation. Proc. Natl Acad. Sci. USA 111, 9265-9270 (2014). 
36. O'Hare, H. M. et al. Regulation of glutamate metabolism by protein kinases in mycobacteria. Mol. Microbiol. 70, 1408-1423 (2008).

37. Khan, M. Z. et al. Protein kinase G confers survival advantage to Mycobacterium tuberculosis during latency-like conditions. J. Biol. Chem. 292, 16093-16108 (2017)

38. Zhang, C. C. Bacterial signalling involving eukaryotic-type protein kinases. Mol. Microbiol. 20, 9-15 (1996).

39. Ortiz-Lombardia, M., Pompeo, F., Boitel, B. \& Alzari, P. M. Crystal structure of the catalytic domain of the $\mathrm{PknB}$ serine/threonine kinase from Mycobacterium tuberculosis. J. Biol. Chem. 278, 13094-13100 (2003).

40. Yeats, C., Finn, R. D. \& Bateman, A. The PASTA domain: a beta-lactambinding domain. Trends Biochem. Sci. 27, 438 (2002).

41. Shah, I. M., Laaberki, M. H., Popham, D. L. \& Dworkin, J. A eukaryotic-like Ser/Thr kinase signals bacteria to exit dormancy in response to peptidoglycan fragments. Cell 135, 486-496 (2008).

42. Righino, B. et al. Structural model of the full-length Ser/Thr protein kinase StkP from S. pneumoniae and its recognition of peptidoglycan fragments. J. Biomol. Struct. Dyn. 7, 1-14 (2017).

43. Turapov, O. et al. The external PASTA domain of the essential serine/ threonine protein kinase PknB regulates mycobacterial growth. Open Biol. 5, 150025 (2015).

44. Mahapatra, S., Crick, D. C., McNeil, M. R. \& Brennan, P. J. Unique structural features of the peptidoglycan of Mycobacterium leprae. J. Bacteriol. 190, 655-661 (2008).

45. Gee, C. L. et al. A phosphorylated pseudokinase complex controls cell wall synthesis in mycobacteria. Sci. Signal. 5, ra7 (2012).

46. Turapov, O. et al. Two faces of CwlM, an essential PknB substrate, in Mycobacterium tuberculosis. Cell Rep. 25, 57-67 e55 (2018).

47. Pompeo, F., Foulquier, E., Serrano, B., Grangeasse, C. \& Galinier, A. Phosphorylation of the cell division protein GpsB regulates PrkC kinase activity through a negative feedback loop in Bacillus subtilis. Mol. Microbiol. 97, 139-150 (2015).

48. Khan, S. et al. Phosphorylation of enoyl-acyl carrier protein reductase InhA impacts mycobacterial growth and survival. J. Biol. Chem. 285, 37860-37871 (2010).

49. Molle, V. et al. Phosphorylation of InhA inhibits mycolic acid biosynthesis and growth of Mycobacterium tuberculosis. Mol. Microbiol. 78, 1591-1605 (2010)

50. Vilcheze, C. et al. Phosphorylation of KasB regulates virulence and acidfastness in Mycobacterium tuberculosis. PLoS Pathog. 10, e1004115 (2014).

51. Corrales, R. M. et al. Phosphorylation of mycobacterial PcaA inhibits mycolic acid cyclopropanation: consequences for intracellular survival and for phagosome maturation block. J. Biol. Chem. 287, 26187-26199 (2012).

52. Pandey, A. K. et al. Nitrile-inducible gene expression in mycobacteria. Tuberculosis (Edinb.) 89, 12-16 (2009).

53. Nagarajan, S. N. et al. Protein kinase A (PknA) of Mycobacterium tuberculosis is independently activated and is critical for growth in vitro and survival of the pathogen in the host. J. Biol. Chem. 290, 9626-9645 (2015).

54. Puri, R. V., Reddy, P. V. \& Tyagi, A. K. Secreted acid phosphatase (SapM) of Mycobacterium tuberculosis is indispensable for arresting phagosomal maturation and growth of the pathogen in guinea pig tissues. PLOS ONE $\mathbf{8}$ e70514 (2013)

55. Dominguez, C., Boelens, R. \& Bonvin, A. M. HADDOCK: a protein-protein docking approach based on biochemical or biophysical information. J. Am. Chem. Soc. 125, 1731-1737 (2003).

56. de Vries, S. J. et al. HADDOCK versus HADDOCK: new features and performance of HADDOCK2.0 on the CAPRI targets. Proteins 69, 726-733 (2007).

57. Morris, G. M. et al. Automated docking using a Lamarckian genetic algorithm and and empirical binding free energy function. J. Comput. Chem. 19, 1639-1662 (1998).

58. O’Boyle, N. M. et al. Open Babel: an open chemical toolbox. J. Cheminform. 3, 33 (2011).

59. Jain, R., Watson, U., Vasudevan, L. \& Saini, D. K. ERK activation pathways downstream of GPCRs. Int. Rev. Cell Mol. Biol. 338, 79-109 (2018).
60. Schneider, C. A., Rasband, W. S. \& Eliceiri, K. W. NIH Image to ImageJ: 25 years of image analysis. Nat. Methods 9, 671-675 (2012).

61. Dephoure, N. \& Gygi, S. P. A solid phase extraction-based platform for rapid phosphoproteomic analysis. Methods 54, 379-386 (2011).

62. Shaik, A. A. et al. Phosphoprotein network analysis of white adipose tissues unveils deregulated pathways in response to high-fat diet. Sci. Rep. 6, 25844 (2016).

63. Oliveros, J. C. VENNY. An interactive tool for comparing lists with Venn's diagrams (2007-2015)

64. Vizcaino, J. A. et al. 2016 update of the PRIDE database and its related tools. Nucleic Acids Res. 44, D447-D456 (2016).

\section{Acknowledgements}

This work was supported by the funding provided by Department of Biotechnology, Government of India (DST/INT/TUNISIA/P-17/2017 \& BT/PR13522/COE/34/27/2015) to V.K.N.; P.K. is a Senior Research Fellow. We thank Dr. Francesca Forti for kindly gifting $R v \Delta B$ conditional mutant. We thank the Central Mass Spec facility of at NII and Mrs. Shanta Sen for her support in managing the facility. We thank Dr. Swati Saha for critical reading of the manuscript. We are grateful to Dr. Sudeepa Rajan for her help with CD experiments and Dr. Savita Lochab, Dr. Mansoor Hussain for their assistance in microscopy.

\section{Author contributions}

P.K., M.R., B.M., U.W., N.P.D., Y.C., S.S., and K.S. were involved in execution of experiments, data acquisition, analysis, and providing raw data for figures. T.S., G.D.J., D.S., D.M., F.G., and V.K.N. provided the scientific overview. P.K. and V.K.N. were involved in overall experimental design, manuscript writing, and making figures. V.K.N. guided the study.

\section{Additional information}

Supplementary Information accompanies this paper at https://doi.org/10.1038/s41467 019-09223-9.

Competing interests: The authors declare no competing interests.

Reprints and permission information is available online at http://npg.nature.com/ reprintsandpermissions/

Journal peer review information: Nature Communications thanks Nelson da Cruz Soares and Robert Husson for their contribution to the peer review of this work. Peer reviewer reports are available.

Publisher's note: Springer Nature remains neutral with regard to jurisdictional claims in published maps and institutional affiliations.

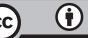

Open Access This article is licensed under a Creative Commons Attribution 4.0 International License, which permits use, sharing, adaptation, distribution and reproduction in any medium or format, as long as you give appropriate credit to the original author(s) and the source, provide a link to the Creative Commons license, and indicate if changes were made. The images or other third party material in this article are included in the article's Creative Commons license, unless indicated otherwise in a credit line to the material. If material is not included in the article's Creative Commons license and your intended use is not permitted by statutory regulation or exceeds the permitted use, you will need to obtain permission directly from the copyright holder. To view a copy of this license, visit http://creativecommons.org/ licenses/by/4.0/

(c) The Author(s) 2019 